\title{
HOMOCLINIC ORBITS FOR SECOND ORDER HAMILTONIAN SYSTEMS POSSESSING SUPERQUADRATIC POTENTIALS
}

\author{
VITTORIO COTI ZELATI AND PAUL H. RABINOWITZ
}

\section{INTRODUCTION}

The goal of this paper is to establish the existence of infinitely many homoclinic orbits for a class of second order Hamiltonian systems of the form:

$$
\ddot{q}-L(t) q+V_{q}(t, q)=0 .
$$

Here $q \in \mathbf{R}^{n}$, and we assume the $n \times n$ matrix $L(t)$ satisfies

$$
L(t) \in C\left(\mathbf{R}, \mathbf{R}^{n^{2}}\right),
$$

is $T$-periodic in $t$, and is symmetric and positive definite uniformly for $t \in$ $[0, T]$. The function $V$ satisfies

$\left(\mathrm{V}_{1}\right) \quad V \in C^{2}\left(\mathbf{R} \times \mathbf{R}^{n}, \mathbf{R}\right)$ and $V(t, q)$ is $T$-periodic in $t$, $\left(\mathrm{V}_{2}\right) \quad V_{q q}(t, 0)=0$

$\left(\mathrm{V}_{3}\right)$ There is a $\mu>2$ such that

$$
0<\mu V(t, q) \leq q \cdot V_{q}(t, q) \text { for all } q \in \mathbf{R}^{n} \backslash\{0\} .
$$

Integrating $\left(\mathrm{V}_{3}\right)$ shows $V(t, q)=o\left(|q|^{2}\right)$ as $|q| \rightarrow 0$ and $V(t, q)|q|^{-2} \rightarrow \infty$ as $|q| \rightarrow \infty$, i.e., $V$ is a "superquadratic" potential.

Our approach to (HS) involves the use of variational methods of a mini-max nature. To describe them more fully, let $E=W^{1,2}\left(\mathbf{R}, \mathbf{R}^{n}\right)$ under the usual norm

$$
\left(\int_{-\infty}^{\infty}\left(|\dot{q}|^{2}+|q|^{2}\right) d t\right)^{1 / 2} .
$$

Thus $E$ is a Hilbert space and it is not difficult to show that $E \subset C^{0}\left(\mathbf{R}, \mathbf{R}^{n}\right)$, the space of continuous functions $q$ on $\mathbf{R}$ such that $q(t) \rightarrow 0$ as $|t| \rightarrow \infty$ (see,

Received by the editors November 27, 1990.

1991 Mathematics Subject Classification. Primary 34C99, 58E99, 58F99.

The first author was supported by Ministero P. I. gruppo 40\% "Calcolo delle variazioni... ".

The second author's research was sponsored in part by the U.S. Army Research Office under contract \#DAAL03-87-K-0043, the National Science Foundation under Grant \#MCS-8110556 and the Office of Naval Research under Grant \#N00014-88-K0134. Any reproduction for the purpose of the United States Government is permitted. 
e.g., [1]). We will seek solutions of (HS) as critical points of the functional $I$ associated with (HS) and given by

$$
I(q)=\int_{-\infty}^{\infty}\left[\frac{1}{2}\left(|\dot{q}|^{2}+L(t) q \cdot q\right)-V(t, q)\right] d t .
$$

By $(\mathrm{L})$,

$$
\|q\|^{2}=\int_{-\infty}^{\infty}\left(|\dot{q}|^{2}+L(t) q \cdot q\right) d t
$$

can and will be taken as an equivalent norm on $E$. Hence $I$ can be written as

$$
I(q)=\frac{1}{2}\|q\|^{2}-\int_{-\infty}^{\infty} V(t, q) d t
$$

As is shown in $\S 1,\left(\mathrm{~V}_{1}\right)-\left(\mathrm{V}_{3}\right)$ imply that $I \in C^{1}(E, \mathbf{R})$. (In fact $I \in$ $C^{2}(E, \mathbf{R})$ but this fact will not be used.) Moreover, critical points of $I$ are classical solutions of (HS) satisfying $\dot{q}(t) \rightarrow 0$ as $|t| \rightarrow \infty$. Thus $q$ is a homoclinic solution of (HS). Note also that $I$ possesses a $\mathbf{Z}$-action. If $q \in E, j \in \mathbf{Z}$, and $\tau_{j}(q)=q(t-j T)$, then

$$
I\left(\tau_{j} q\right)=I(q)
$$

To prove the existence of critical points of functionals like (0.2), especially multiple critical points, one generally needs some compactness as embodied by the Palais-Smale condition (PS) or one of its variants. (PS) says whenever $I\left(u_{m}\right)$ is bounded and $I^{\prime}\left(u_{m}\right) \rightarrow 0$ as $m \rightarrow \infty$, the sequence $u_{m}$ possesses a convergent subsequence. Unfortunately (PS) does not hold for $(0.2)$ even if we divide out the $\mathbf{Z}$ symmetry. Indeed suppose $b$ is a critical value of $I$ with corresponding critical point $q$. Consider $u_{m}=q+\tau_{m} q$. Then $I\left(u_{m}\right) \rightarrow 2 b$ and $I^{\prime}\left(u_{m}\right) \rightarrow 0$ as $m \rightarrow \infty$. Thus (PS) fails at $2 b$ and similarly at $k b$ for all $k \in \mathbf{N} \backslash\{1\}$.

Let $I^{s} \equiv\{q \in E \mid I(q) \leq s\}, I_{s}=\{q \in E \mid I(q) \geq s\}$, and $I_{b}^{a}=I^{a} \cap I_{b}$. As will be shown in $\S 1$, hypotheses $\left(\mathrm{V}_{1}\right)-\left(\mathrm{V}_{3}\right)$ imply $I(q)=\frac{1}{2}\|q\|^{2}+o\left(\|q\|^{2}\right)$ as $q \rightarrow 0$ and $I^{0} \backslash\{0\} \neq \varnothing$. If (PS) were satisfied these facts together with the Mountain Pass Theorem would imply that $I$ has a critical value $c>0$ given by

$$
c=\inf _{g \in \Gamma} \max _{\theta \in[0,1]} I(g(\theta))
$$

where

$$
\Gamma=\left\{g \in C([0,1], E) \mid g(0)=0 \text { and } g(1) \in I^{0} \backslash\{0\}\right\} .
$$

The number $c$ in (0.4) plays a special role in our study of (HS). We will prove:

Theorem 0.5. If $\left(\mathrm{V}_{1}\right)-\left(\mathrm{V}_{3}\right)$ and $(\mathrm{L})$ are satisfied and

(*) there is an $\alpha>0$ such that $I^{c+a} / \mathbf{Z}$ contains only finitely many critical points of $I$, 
then $c$ is a critical value of $I$. Moreover for each $k \in \mathbf{N} \backslash\{1\}, I_{k c-\alpha}^{k c+\alpha} / \mathbf{Z}$ contains infinitely many distinct critical points of $I$.

As noted above, each of these critical points is then a classical homoclinic solution of (HS). Of course if $(*)$ is not satisfied then $I$ already has infinitely many distinct critical points in $I^{c+\alpha} / \mathbf{Z}$. Our main result, Theorem 3.34 , is a more precise version of Theorem 0.5 and tells us there are critical points of $I$ of a certain form. Roughly speaking it says if $q$ is a mountain pass critical point, i.e., $I(q)=c$ and $I^{\prime}(q)=0$, then there is a critical point of $I$ near $\sum_{j=1}^{k} \tau_{m_{j}} q$ for all $\left(m_{1}, \ldots, m_{k}\right) \in \mathbf{Z}^{k}$ provided that $\left(m_{i}-m_{j}\right)$ is sufficiently large for all $i \neq j$. Alternatively interpreting the main result from the point of view of dynamical systems, it roughly says for each $k \in \mathbf{N} \backslash\{1\}$, there is a homoclinic solution of (HS) which emanates from 0 at $t=-\infty$, returns to a neighborhood of 0 and spends at least a prescribed amount of time there, and repeats this process $k-1$ more times before terminating at $t=\infty$. Thus our approach to (HS) is a kind of variational version of shadowing.

Observe that if $\left(\mathrm{V}_{1}\right)-\left(\mathrm{V}_{3}\right)$ and $(\mathrm{L})$ are satisfied and $V$ and $L$ are independent of $t$, then (0.3) holds for all $j \in \mathbf{R}$. In particular, if $q \in I^{c+\alpha} \backslash\{0\}$ is a solution of (HS) (and the existence of such a $q \in I^{c}$ was established in [1]), then for $j \in[0,1)$ the functions $\tau_{j} q$ are distinct solutions of (HS) in $I^{c+\alpha}$. Therefore $(*)$ is not satisfied in the autonomous case. Indeed there may only be one homoclinic solution of (HS) (up to translation) for the autonomous case (as can be easily seen in the case of $n=1$, e.g., $L(t) \equiv 1, V(t, q) \equiv q^{4}$ for $q \geq 0$ and $\equiv q^{3}$ for $q<0$ ) and it is essential for our results that $L$ or $V$ depend explicitly on $t$.

One final remark about $(*)$ is in order. In the usual dynamical systems approach to homoclinics, given one homoclinic solution and an associated Poincaré map for the equation for which the stable and unstable manifolds intersect transversally at the corresponding homoclinic point, one gets a rich structure of homoclinics and other special solutions of the equation nearby. It is generally difficult to verify such a transversal intersection condition. In some sense $(*)$ replaces this condition in our setting. However it does not seem to be easy to make a direct comparison of these two conditions.

A precise statement of the main result will be given in $\S 3$. In $\S \S 1$ and 2 several technical results will be proved. In particular the behavior of (PS) sequences will be analyzed in $\S 1$ and a "Deformation" Theorem will be proved in $\S 2$. Then the main result will be stated and proved in $\S \S 3$ and 4 . Lastly a stronger result under an additional condition will be discussed in $\S 5$.

There have only been a few papers written which use variational methods to find homoclinic solutions of Hamiltonian systems [1-5]. In [2], V. Coti Zelati, Ekeland, and Séré studied a general Hamiltonian system of the form

$$
\dot{z}=\mathscr{J}\left(A z+\widehat{H}_{z}(t, z)\right) \text {. }
$$

Here $z \in \mathbf{R}^{2 n}, \mathscr{J}$ is the usual symplectic matrix, and $A$ is a hyperbolic 
matrix. The function $\widehat{H}$ is $T$-periodic in $t$, convex in $z, o\left(|z|^{2}\right)$ as $|z| \rightarrow 0$, and satisfies an analogue of $\left(\mathrm{V}_{3}\right)$ as well as a suitable growth condition. Using a dual variational transformation, arguments involving understanding the behavior of (PS) sequences, the Mountain Pass Theorem, and another mini-max argument, the existence of two distinct homoclinic orbits is proved. Next the paper [1] studied (HS) under slightly weaker conditions than $\left(\mathrm{V}_{1}\right)-\left(\mathrm{V}_{3}\right)$ and proved the existence of one homoclinic solution which moreover was the limit of subharmonic solutions, $q_{j}$ (i.e., $2 j T$-periodic solutions), as $j \rightarrow \infty$. This was done using the Mountain Pass Theorem to get the subharmonic solutions and appropriate estimates to pass to a nontrivial limit. Lastly in [3], which is the first work we know of on the existence of infinitely many homoclinics using variational methods, Séré extended the results of [2] to prove that there exist infinitely many distinct homoclinics. He assumes condition $(*)$ where $c$ is his analogue of $(0.4)$. We have benefited from some of the ideas in $[2,3]$.

Finally we note that extensions have been made of the results of Coti Zelati, Ekeland, and Séré [2] by Hofer and Wysocki [4] and Tanaka [5]. These generalizations use different arguments than those of [2] to establish the existence of one homoclinic solution.

\section{SOME PRELIMINARIES}

In this section, some of the preliminaries concerning the properties of $I$ that will be needed later will be carried out. Even if not explicitly stated, except for Proposition 1.1 below, we always assume $V$ satisfies $\left(\mathrm{V}_{1}\right)-\left(\mathrm{V}_{3}\right)$. With $E$ and $I$ as defined in the Introduction we have

Proposition 1.1. If $V$ satisfies $\left(\mathrm{V}_{1}\right)-\left(\mathrm{V}_{2}\right)$, then $I \in C^{1}(E, \mathbf{R})$.

Proof. First we show $I: E \rightarrow \mathbf{R}$. By $\left(\mathrm{V}_{1}\right)-\left(\mathrm{V}_{2}\right)$, there is a $\delta>0$ such that $|x| \leq \delta$ implies

$$
V(t, x) \leq|x|^{2} .
$$

Let $q \in E$. Then $q \in C^{0}\left(\mathbf{R}, \mathbf{R}^{n}\right)$ [1]. Therefore there is an $R \geq 0$ such that $|t|>R$ implies $|q(t)| \leq \delta$. Hence by (1.2),

$$
\int_{-\infty}^{\infty} V(t, q) d t \leq \int_{-R}^{R} V(t, q) d t+\int_{|t|>R}|q|^{2} d t<\infty
$$

and $I: E \rightarrow \mathbf{R}$.

Next we prove $I$ is Fréchet differentiable on $E$. It suffices to show this is the case for

$$
J(q)=\int_{-\infty}^{\infty} V(t, q) d t
$$

In the process we will see that

$$
J^{\prime}(q) \varphi=\int_{-\infty}^{\infty} V_{q}(t, q) \cdot \varphi d t
$$


and this is defined for all $q, \varphi \in E$. Let $q \in E$ and $\varepsilon>0$. Suppose $\|q\|=M$. Using $\left(\mathrm{V}_{1}\right)-\left(\mathrm{V}_{2}\right)$, choose $\rho>0$ such that $|x| \leq \rho$ implies

$$
\left|V_{q}(t, x)\right| \leq \frac{\varepsilon}{4(M+1)}|x| .
$$

It is known (see,e.g., [6]) that

$$
\int_{-R}^{R} V(t, q) d t \in C^{1}\left(W^{1,2}\left([-R, R], \mathbf{R}^{n}\right) ; \mathbf{R}\right)
$$

for any finite $R$. Therefore there is a $\delta=\delta(\varepsilon, R, q)<\min (\rho / 4,1)$ such that $\varphi \in E$ and $\|\varphi\| \leq \delta$ implies

$$
\left|\int_{-R}^{R}\left(V(t, q+\varphi)-V(t, q)-V_{q}(t, q) \cdot \varphi\right) d t\right| \leq \frac{\varepsilon}{4}\|\varphi\| .
$$

Choose $R$ so large that $|q(t)| \leq \rho / 4$ for $|t| \geq R$. For $\varphi \in E$,

$$
\|\varphi\|_{L^{\infty}\left(\mathbf{R}, \mathbf{R}^{n}\right)} \leq \sqrt{2}\|\varphi\| .
$$

Therefore

$$
\|\varphi\|_{L^{\infty}} \leq \rho / 2 .
$$

The Mean Value Theorem, (1.4), and (1.8) show for $|t| \geq R$,

$$
|V(t, q+\varphi)-V(t, q)| \leq \frac{\varepsilon(|q|+|\varphi|)}{4(M+1)}|\varphi| .
$$

Hence

$$
\begin{aligned}
\int_{|t|>R}|V(t, q+\varphi)-V(t, q)| d t & \leq \frac{\varepsilon}{4(M+1)}\left(\int_{|t|>R}(|q|+|\varphi|)^{2} d t\right)^{1 / 2}\|\varphi\| \\
& \leq \frac{\varepsilon}{4}\|\varphi\| .
\end{aligned}
$$

Likewise, by (1.4)

$$
\int_{|t|>R}\left|V_{q}(t, q) \varphi\right| d t \leq \frac{\varepsilon}{4(M+1)} \int_{|t|>R}|q||\varphi| d t \leq \frac{\varepsilon}{4}\|\varphi\| .
$$

Combining (1.6), (1.10), and (1.11) yields the Fréchet differentiability of $J$.

Lastly to prove that $J^{\prime}$ is continuous, suppose $q_{m} \rightarrow q$ in $E$ (and therefore in $L^{\infty}$ ). Note that

$$
\begin{aligned}
& \sup _{\|\varphi\|=1}\left|\int_{-\infty}^{\infty}\left(V_{q}\left(t, q_{m}\right)-V_{q}(t, q)\right) \cdot \varphi d t\right| \\
& \quad \leq\left(\int_{-\infty}^{\infty}\left|V_{q}\left(t, q_{m}\right)-V_{q}(t, q)\right|^{2} d t\right)^{1 / 2} .
\end{aligned}
$$

Let $\varepsilon>0$. Choose $R$ so that $|t| \geq R$ implies

$$
\left|V_{q}(t, q)\right| \leq \frac{\varepsilon}{2}|q| .
$$


We can also assume (1.13) holds for $q_{m}$ for large $m$. Therefore by (1.12)(1.13)

$$
\begin{aligned}
& \left(\int_{-\infty}^{\infty}\left|V_{q}\left(t, q_{m}\right)-V_{q}(t, q)\right|^{2} d t\right)^{1 / 2} \\
& \quad \leq\left(\int_{-R}^{R}\left|V_{q}\left(t, q_{m}\right)-V_{q}(t, q)\right|^{2} d t\right)^{1 / 2}+\frac{\varepsilon}{2}\left(\left\|q_{m}\right\|_{L^{2}}+\|q\|_{L^{2}}\right)
\end{aligned}
$$

which implies the continuity of $J^{\prime}$.

Remark 1.15. Since $V \in C^{2}$ by $\left(\mathrm{V}_{1}\right)$, it can be shown that $I \in C^{2}(E, \mathbf{R})$. However we will make no use of this fact.

Lemma 1.16. If $V$ satisfies $\left(\mathrm{V}_{1}\right)-\left(\mathrm{V}_{2}\right)$, then

$$
I(q)=\frac{1}{2}\|q\|^{2}+o\left(\|q\|^{2}\right) \text { as } q \rightarrow 0 .
$$

Proof. It suffices to show $J(q)=o\left(\|q\|^{2}\right)$ as $q \rightarrow 0$. Let $\varepsilon>0$. By $\left(\mathrm{V}_{1}\right)-\left(\mathrm{V}_{2}\right)$, there is a $\rho>0$ such that $|x| \leq \rho$ implies

$$
|V(t, x)| \leq \varepsilon|x|^{2}
$$

Choose $\delta=\rho / 2$. Hence, by (1.7), $\|q\| \leq \delta$ implies $\|q\|_{L^{\infty}} \leq \sqrt{2}\|q\| \leq \rho$. Thus, by (1.17),

$$
J(q) \leq \varepsilon \int_{-\infty}^{\infty}|q|^{2} d t \leq \varepsilon\|q\|^{2} .
$$

Remark 1.19. Let $\mathscr{K}$ denote the set of critical points of $I$. Lemma 1.16 implies 0 is an isolated point in $\mathscr{K}$. Therefore there exists a $\nu>0$ such that $x \in \mathscr{K} \backslash\{0\}$ implies $\|x\| \geq \nu$.

Remark 1.20. Let $y \in E \backslash\{0\}$. Then $\left(\mathrm{V}_{1}\right)$ and $\left(\mathrm{V}_{3}\right)$ imply that $I(\beta y) \rightarrow-\infty$ as $|\beta| \rightarrow \infty$ (see, e.g., [1]). Thus $I^{0} \backslash\{0\} \neq \varnothing$. This observation together with Proposition 1.1 and Lemma 1.16 shows that $I$ satisfies the hypotheses of the Mountain Pass Theorem aside from the Palais-Smale condition (PS). Unfortunately (PS) does not hold. However it was shown in [1] that (HS) possesses a nontrivial homoclinic solution under milder hypothesis than $\left(V_{1}\right)-\left(V_{3}\right)$.

We will study the behavior of (PS) sequences. First we need some estimates.

Lemam 1.21. Let $V$ satisfy $\left(\mathrm{V}_{1}\right)-\left(\mathrm{V}_{3}\right)$. Then

(i) There is a $\underline{c}>0$ such that $I(q) \geq \underline{c}$ for all $q \in \mathscr{K} \backslash\{0\}$.

(ii) If $I(q)=b$ and $q \in \mathscr{K} \backslash\{0\}$,

$$
\|q\| \leq\left(\frac{2 \mu b}{\mu-2}\right)^{1 / 2} .
$$


Proof. Note first that (ii) and Remark 1.19 imply (i). To prove (ii), observe that

$$
\begin{aligned}
b & =I(q)=I(q)-\frac{1}{2} I^{\prime}(q) q=\int_{-\infty}^{\infty}\left[\frac{1}{2} q \cdot V_{q}(t, q)-V(t, q)\right] d t \\
& \geq\left(\frac{1}{2}-\frac{1}{\mu}\right) \int_{-\infty}^{\infty} q \cdot V_{q}(t, q) d t>0
\end{aligned}
$$

if $q \neq 0$ via $\left(\mathrm{V}_{3}\right)$. Since (HS) holds for $q \in \mathscr{K}$, multiplying (HS) by $q$ and integrating by parts shows

$$
\|q\|^{2}=\int_{-\infty}^{\infty} q \cdot V_{q}(t, q) d t
$$

Thus (ii) follows from (1.22)-(1.23).

Now the behavior of (PS) sequences, i.e., sequences such that $I\left(u_{m}\right) \rightarrow b$ and $I^{\prime}\left(u_{m}\right) \rightarrow 0$, will be studied. We obtain a result like that of [2] by a somewhat more direct argument. As was observed in the Introduction, if $v \in \mathscr{K} \backslash\{0\}$, so is $\tau_{j} v$ for all $j \in \mathbf{Z}$. The function $v$ can be made unique within the class of translates by requiring that

$$
\|v\|_{L^{\infty}}=\max _{t \in \mathbf{R}}|v(t)|
$$

occurs for $t \in[0, T]$ and $|v(t)|<\|v\|_{L^{\infty}}$ for $t<0$. We refer to such a $v \in \mathscr{K} \backslash\{0\}$ as a normalized $v$. In fact any $v \in E \backslash\{0\}$ can be so normalized. Alternatively we could take the normalized $v$ as the representative for the equivalence class of $v$ in $E / \mathbf{Z}$.

Proposition 1.24. Let $\left(u_{m}\right) \subset E$ be such that $I\left(u_{m}\right) \rightarrow b>0$ and $I^{\prime}\left(u_{m}\right) \rightarrow 0$. Then there is an $l \in \mathbf{N}$ with $l$ bounded above by a constant depending only on $b$, normalized functions $v_{1}, \ldots, v_{l} \in \mathscr{K} \backslash\{0\}$, a subsequence of $u_{m}$, and corresponding $\left(k_{m}^{i}\right) \subset \mathbf{Z}, 1 \leq i \leq l$, such that

$$
\begin{gathered}
\left\|u_{m}-\sum_{1}^{l} \tau_{k_{m}^{i}} v_{i}\right\| \rightarrow 0, \\
\sum_{1}^{l} I\left(v_{i}\right)=b,
\end{gathered}
$$

and, for $i \neq j$,

$$
\left|k_{m}^{i}-k_{m}^{j}\right| \rightarrow \infty
$$

as $m \rightarrow \infty$ along the subsequence. 
Proof. For $m$ large, by $\left(\mathrm{V}_{3}\right)$,

$$
\begin{aligned}
b+\frac{1}{\mu} & \left\|u_{m}\right\| \geq I\left(u_{m}\right)-\frac{1}{\mu} I^{\prime}\left(u_{m}\right) u_{m} \\
& =\left(\frac{1}{2}-\frac{1}{\mu}\right)\left\|u_{m}\right\|^{2}+\int_{-\infty}^{\infty}\left[\frac{1}{\mu} V_{q}\left(t, u_{m}\right) \cdot u_{m}-V\left(t, u_{m}\right)\right] d t \\
& \geq\left(\frac{1}{2}-\frac{1}{\mu}\right)\left\|u_{m}\right\|^{2}
\end{aligned}
$$

Hence $\left(u_{m}\right)$ is bounded in $E$. There exists a unique $p_{m}^{1} \in \mathbf{Z}$ and normalized $u_{m}^{1} \in E$ such that $u_{m}=\tau_{p_{m}^{1}} u_{m}^{1}$. Note that

$$
\left\{\begin{array}{l}
I\left(u_{m}\right)=I\left(u_{m}^{1}\right), \\
\left\|I^{\prime}\left(u_{m}\right)\right\|=\left\|I^{\prime}\left(u_{m}^{1}\right)\right\|, \\
\left\|u_{m}\right\|=\left\|u_{m}^{1}\right\| .
\end{array}\right.
$$

Hence $\left(u_{m}^{1}\right)$ is bounded in $E$ and there is a subsequence of $\left(u_{m}^{1}\right)$ which converges to $u^{1} \in E$ both weakly in $E$ and in $L_{\text {loc }}^{\infty}\left(\mathbf{R}, \mathbf{R}^{n}\right)$. For convenience we assume this is the case for the entire sequence.

We claim $u^{1} \neq 0$. Otherwise $\left\|u_{m}^{1}\right\|_{L^{\infty}} \rightarrow 0$ since $u_{m}^{1} \rightarrow u^{1}$ in $L_{\text {loc }}^{\infty}$ and $\max \left|u_{m}^{1}\right|$ occurs in $[0, T)$. Therefore, by $\left(\mathrm{V}_{2}\right)$, for any $\varepsilon>0$ and $m=m(\varepsilon)$ large, we have

$$
\left\{\begin{array}{l}
\left|V\left(t, u_{m}^{1}\right)\right| \leq \varepsilon\left|u_{m}^{1}\right|^{2}, \\
\left|u_{m}^{1} \cdot V_{q}\left(t, u_{m}^{1}\right)\right| \leq \varepsilon\left|u_{m}^{1}\right|^{2}
\end{array}\right.
$$

and

$$
\begin{aligned}
I\left(u_{m}^{1}\right)-\frac{1}{2} I^{\prime}\left(u_{m}^{1}\right) u_{m}^{1} & =\int_{-\infty}^{\infty}\left[\frac{1}{2} u_{m}^{1} \cdot V_{q}\left(t, u_{m}^{1}\right)-V\left(t, u_{m}^{1}\right)\right] d t \\
& \leq \frac{3}{2} \varepsilon\left\|u_{m}^{1}\right\|_{L^{2}}^{2} \leq \frac{3}{2} \varepsilon\left\|u_{m}^{1}\right\|^{2} .
\end{aligned}
$$

Since $\varepsilon$ is arbitrary, the boundedness of $\left(u_{m}^{1}\right)$ and (1.31) contradict that $I\left(u_{m}^{1}\right)$ $\rightarrow b>0$. Hence $u^{1} \neq 0$.

To see that $u^{1} \in \mathscr{K}$, let $\varphi \in E$ and let $(\cdot, \cdot)$ denote the inner product in $E$. Since

$$
I^{\prime}\left(u^{1}\right) \varphi-I^{\prime}\left(u_{m}^{1}\right) \varphi=\left(u^{1}-u_{m}^{1}, \varphi\right)-\int_{-\infty}^{\infty}\left(V_{q}\left(t, u^{1}\right)-V_{q}\left(t, u_{m}^{1}\right)\right) \cdot \varphi d t
$$

$u_{m}^{1}-u^{1}$, and $I^{\prime}\left(u_{m}^{1}\right) \rightarrow 0$, it follows that $u^{1}$ is a critical point if

$$
\int_{-\infty}^{\infty}\left(V_{q}\left(t, u^{1}\right)-V_{q}\left(t, u_{m}^{1}\right)\right) \cdot \varphi d t \rightarrow 0
$$

as $m \rightarrow \infty$. Let $\varepsilon>0$ and $\bar{T}=\bar{T}(\varepsilon, \varphi)$ be such that

$$
\int_{t \geq \bar{T}}\left(|\dot{\varphi}|^{2}+(L(t) \varphi, \varphi)\right) \leq \varepsilon
$$


Now

$$
\int_{-\infty}^{\infty}\left(V_{q}\left(t, u^{1}\right)-V_{q}\left(t, u_{m}^{1}\right)\right) \cdot \varphi d t=\int_{-\bar{T}}^{\bar{T}} \cdots+\int_{|t|>\bar{T}} \cdots
$$

The first term on the right-hand side of (1.35) approaches 0 as $m \rightarrow \infty$ since $u_{m}^{1} \rightarrow u^{1}$ in $L_{\text {loc }}^{\infty}$. The second term can be estimated by

$$
\begin{aligned}
& \left|\int_{|t| \geq \bar{T}}\left(V_{q}\left(t, u^{1}\right)-V_{q}\left(t, u_{m}^{1}\right)\right) \cdot \varphi d t\right| \\
& \quad \leq\left(\int_{|t| \geq \bar{T}}\left|V_{q}\left(t, u^{1}\right)-V_{q}\left(t, u_{n}^{1}\right)\right|^{2}\right)^{1 / 2} \varepsilon \leq M \varepsilon,
\end{aligned}
$$

where the constant $M$ depends on $V_{q}$ and the bounds for $u_{m}^{1}$ and $u^{1}$ in $E$. Thus letting $m \rightarrow \infty$ in (1.36) and using that $\varphi$ is arbitrary shows that $I^{\prime}\left(u^{1}\right)=0$. Moreover by Lemma 1.21 ,

$$
I\left(u^{1}\right) \geq \underline{c}>0 .
$$

Next let $u_{m}^{2}=u_{m}^{1}-u^{1}$. We claim that

$$
I\left(u_{m}^{2}\right) \rightarrow b-I\left(u^{1}\right)
$$

and

$$
I^{\prime}\left(u_{m}^{2}\right) \rightarrow 0
$$

Indeed

$$
\begin{aligned}
I\left(u_{m}^{1}\right)=I\left(u_{m}^{2}+u^{1}\right)= & I\left(u_{m}^{2}\right)+I\left(u^{1}\right)+\left(u_{m}^{2}, u^{1}\right) \\
& -\int_{-\infty}^{\infty}\left[V\left(t, u_{m}^{2}+u^{1}\right)-V\left(t, u_{m}^{2}\right)-V\left(t, u^{1}\right)\right] d t .
\end{aligned}
$$

Since $\left(u_{m}^{2}, u^{1}\right) \rightarrow 0$ and $I\left(u_{m}^{1}\right) \rightarrow b$ as $m \rightarrow \infty,(1.40)$ shows (1.38) holds if

$$
\int_{-\infty}^{\infty}\left[V\left(t, u_{m}^{2}+u^{1}\right)-V\left(t, u_{m}^{2}\right)-V\left(t, u^{1}\right)\right] d t \rightarrow 0
$$

as $m \rightarrow \infty$. By the $L_{\text {loc }}^{\infty}$ convergence of $u_{m}^{1}$ to $u^{1}$,

$$
\int_{-R}^{R}\left[V\left(t, u_{m}^{2}+u^{1}\right)-V\left(t, u_{m}^{2}\right)-V\left(t, u^{1}\right)\right] d t \rightarrow 0
$$

as $m \rightarrow \infty$ for any $R>0$. Moreover, for any $\varepsilon>0$, there is an $R_{\varepsilon}>0$ such that

$$
\int_{|t| \geq R_{\varepsilon}}\left|u^{1}\right|^{2} d t \leq \varepsilon^{2}, \quad \int_{|t| \geq R_{\varepsilon}} V\left(t, u^{1}\right) d t \leq \varepsilon .
$$

By the Mean Value Theorem, there exists $\theta(t) \in(0,1)$ such that

$$
V\left(t, u_{m}^{2}+u^{1}\right)-V\left(t, u_{m}^{2}\right)=V_{q}\left(t, u_{m}^{2}+\theta u^{1}\right) u^{1}
$$


By $\left(\mathrm{V}_{1}\right)-\left(\mathrm{V}_{2}\right)$, there is a $\delta>0$ such that

$$
\left|V_{q}(t, z)\right| \leq|z|
$$

for $|z| \leq \delta$. For $\delta \leq|z| \leq M$ with $M$ free for the moment,

$$
\left|V_{q}(t, z)\right| \leq\left(\sup _{|t| \geq R_{\varepsilon},|\xi| \leq M}\left|V_{q}(t, \xi)\right|\right) \frac{|z|}{\delta} \equiv M_{1} \frac{|z|}{\delta} .
$$

Choosing $M$ such that

$$
\left\|u_{m}^{2}\right\|_{L^{\infty}}+\left\|u^{1}\right\|_{L^{\infty}} \leq M
$$

(1.43)-(1.46) show

$$
\begin{aligned}
& \int_{|t| \geq R_{\varepsilon}}\left|V\left(t, u_{m}^{2}+u^{1}\right)-V\left(t, u_{m}^{2}\right)-V\left(t, u^{1}\right)\right| d t \\
& \quad \leq\left(1+\frac{M_{1}}{\delta}\right)\left(\int_{|t| \geq R_{\varepsilon}}\left(\left|u_{m}^{2}\right|+\left|u^{1}\right|^{2}\right) d t\right)^{1 / 2}\left(\int_{|t| \geq R_{\varepsilon}}\left|u^{1}\right|^{2} d t\right)^{1 / 2}+\varepsilon \\
& \quad \leq M_{2} \varepsilon+\varepsilon
\end{aligned}
$$

Since $\varepsilon$ is arbitrary and $M_{2}$ can be chosen independently of $\varepsilon,(1.47)$ and (1.42) imply that (1.41) holds. Thus (1.38) is proved.

To verify (1.39), observe that

$$
I^{\prime}\left(u_{m}^{2}\right) \varphi=I^{\prime}\left(u_{m}^{1}\right) \varphi-\int_{-\infty}^{\infty}\left(V_{q}\left(t, u_{m}^{2}\right)-V_{q}\left(t, u_{m}^{1}\right)+V_{q}\left(t, u^{1}\right)\right) \cdot \varphi d t
$$

Since $I^{\prime}\left(u_{m}^{1}\right) \rightarrow 0$ as $m \rightarrow \infty,(1.39)$ reduces to proving

$$
\sup _{\|\varphi\|=1}\left|\int_{-\infty}^{\infty}\left(V_{q}\left(t, u_{m}^{2}\right)-V_{q}\left(t, u_{m}^{1}\right)+V_{q}\left(t, u^{2}\right)\right) \cdot \varphi d t\right| \rightarrow 0
$$

as $m \rightarrow \infty$. By $\left(\mathrm{V}_{1}\right)-\left(\mathrm{V}_{2}\right)$, there is a $\delta>0$ such that

$$
\left|V_{q}(t, q)\right| \leq|q|
$$

if $|q| \leq \delta$. Let $\varepsilon>0$. Choose $R_{\varepsilon}$ so that $|t| \geq R_{\varepsilon}$ implies

$$
\left|u^{1}(t)\right| \leq \delta, \quad \int_{|t| \geq R_{\varepsilon}}\left|u^{1}\right|^{2} d t<\varepsilon^{2}
$$

Now

$$
\begin{aligned}
& \left|\int_{-\infty}^{\infty}\left(V_{q}\left(t, u_{m}^{2}\right)-V_{q}\left(t, u_{m}^{1}\right)+V_{q}\left(t, u^{1}\right)\right) \cdot \varphi d t\right| \\
& \quad \leq\left|\int_{-R_{\varepsilon}}^{R_{\varepsilon}} \cdots\right|+\left|\int_{t \geq R_{\varepsilon}} \ldots\right| .
\end{aligned}
$$

The first integral on the right-hand side of (1.52) tends to 0 as $m \rightarrow \infty$. Using $\left(\mathrm{V}_{1}\right)-\left(\mathrm{V}_{2}\right)$ again and arguing in a similar way to (1.44)-(1.47) yields

$$
\begin{aligned}
& \left|\int_{|t| \geq R_{\varepsilon}}\left(V_{q}\left(t, u_{m}^{2}\right)-V_{q}\left(t, u_{m}^{1}\right)\right) \cdot \varphi d t\right|+\int_{|t| \geq R_{\varepsilon}}\left|V_{q}\left(t, u^{1}\right) \cdot \varphi\right| d t \\
& \quad \leq(M+1) \int_{|t| \geq R_{\varepsilon}}\left|u^{1} \cdot \varphi\right| d t \leq(M+1) \varepsilon\|\varphi\|
\end{aligned}
$$


where the constant $M$ is independent of $\varepsilon$. Thus (1.53) implies (1.49) and (1.39) is proved.

Note that by the computation of $(1.22), b-I\left(u^{1}\right) \geq 0$. Therefore there are now two possibilities to consider:

(a) Case 1: $b=I\left(u^{1}\right)$. Thus $I\left(u_{m}^{2}\right) \rightarrow 0$ and $I^{\prime}\left(u_{m}^{2}\right) \rightarrow 0$ as $m \rightarrow \infty$. Consequently, by (1.28), $\left\|u_{m}^{2}\right\| \rightarrow 0$ as $m \rightarrow \infty$. But then $\left\|u_{m}^{1}-u^{1}\right\|=\left\|u_{m}-\tau_{p_{m}^{1}} u_{1}\right\| \rightarrow$ 0 . The function $u^{1}$ may not be normalized but there is a $p_{1} \in \mathbf{Z}$ such that $u^{1}=\tau_{p_{1}} v_{1}$ with $v_{1}$ normalized. Thus Proposition 1.24 holds with $l=1$ and $k_{m}^{1}=p_{m}^{1}+p_{1}$.

(b) Case 2: $b>I\left(u^{1}\right)$. Then we are back to our original situation with $u_{m}$ replaced by $u_{m}^{2}$ and $b$ by $b-I\left(u^{1}\right)<b-\underline{c}$. Hence by what was shown above, $u_{m}^{2}=\tau_{p_{m}^{2}} w_{m}^{2}$ with $\left(p_{m}^{2}\right) \subset \mathbf{Z}, w_{m}^{2}$ is normalized, a subsequence of $w_{m}^{2}$ converges weakly in $E$ and in $L_{\text {loc }}^{\infty}$ to $u^{2} \in \mathscr{K} \backslash\{0\}$, and $I\left(w_{m}^{2}-u^{2}\right) \rightarrow$ $b-I\left(u^{1}\right)-I\left(u^{2}\right), I^{\prime}\left(w_{m}^{2}-u^{2}\right) \rightarrow 0$. If $b=I\left(u^{1}\right)+I\left(u^{2}\right)$, then by Case 1

$$
\begin{aligned}
\left\|w_{m}^{2}-u^{2}\right\| & =\left\|\tau_{-p_{m}^{2}} u_{m}^{2}-u^{2}\right\|=\left\|\tau_{-p_{m}^{2}}\left(u_{m}^{1}-u^{1}\right)-u^{2}\right\| \\
& =\left\|u_{m}-\tau_{p_{m}^{1}} u^{1}-\tau_{p_{m}^{2}+p_{m}^{1}} u^{2}\right\| \rightarrow 0 .
\end{aligned}
$$

Note that $\left(p_{m}^{2}\right)$ cannot be bounded since $u_{m}^{2} \rightarrow 0$ while $\tau_{-p_{m}^{2}} u_{m}^{2} \rightarrow u^{2} \epsilon$ $\mathscr{K} \backslash\{0\}$. Thus after normalizing $u^{1}, u^{2}$, we get Proposition 1.24 with $l=2$, $k_{m}^{1}=p_{m}^{1}+p_{1}$, and $k_{m}^{2}=p_{m}^{2}+p_{m}^{1}+p_{2}$. If $b>I\left(u^{1}\right)+I\left(u^{2}\right)$, then continuing in this fashion in at most $[b / c]$ steps, the proposition follows.

Remark 1.54. The only role $\left(\mathrm{V}_{3}\right)$ plays in this proposition is in (1.28) to obtain bounds for $\left\|u_{m}\right\|$ in terms of $b$. Thus the proposition also holds if $\left(\mathrm{V}_{3}\right)$ is replaced by an assumption about the existence of such bounds.

Our final result in this section concerns a discreteness property of the set of sums of translates of $\mathscr{K}^{c+\alpha} / \mathbf{Z}$. We will state it in a more general form.

Proposition 1.55. Let $F \subset E$ be a finite set of points and $l \in \mathbf{N}$. Let

$$
\mathscr{T}_{l}(F)=\left\{\sum_{1}^{j} \tau_{k_{i}} v_{i} \mid 1 \leq j \leq l, v_{i} \in F, k_{i} \in \mathbf{Z}\right\} .
$$

Let $\mu=\mu\left(\mathscr{T}_{l}(F)\right)=\inf \left\{\|x-y\| \mid x \neq y \in \mathscr{T}_{l}(F)\right\}$. Then $\mu>0$.

Proof. Let $\left(x_{m}\right),\left(y_{m}\right) \subset \mathscr{T}_{l}(F)$ such that $x_{m} \neq y_{m}$ and $\left\|x_{m}-y_{m}\right\| \rightarrow \mu$. Then

$$
x_{m}=\sum_{i=1}^{j_{m}} \tau_{k_{m}^{i}} v_{m}^{i}, \quad y_{m}=\sum_{i=1}^{r_{m}} \tau_{p_{m}^{i}} w_{m}^{i},
$$

where $1 \leq j_{m}, r_{m} \leq l$ and $v_{m}^{i}, w_{m}^{i} \in F$. Since $F$ is finite, without loss of generality it can be assumed that $j_{m}, r_{m}, v_{m}^{i}$, and $w_{m}^{i}$ are independent of $m$. 
Therefore

$$
\left\|\sum_{i=1}^{j} \tau_{k_{m}^{i}} v_{i}-\sum_{i=1}^{r} \tau_{p_{m}^{i}} w_{i}\right\| \rightarrow \mu .
$$

Since $\|x\|=\left\|\tau_{j} x\right\|$ for all $x \in E$ and $j \in \mathbf{Z}$, we can assume $k_{m}^{1}=0$. Now two cases will be considered:

Case (i): $\left(k_{m}^{*}\right)$ and $\left(p_{m}^{s}\right)$ are bounded, $1 \leq i \leq j, 1 \leq s \leq r$. Then passing to a subsequence if necessary, it can be assumed that $k_{m}^{i}=k_{i}$ and $p_{m}^{s}=p_{s}$ for large $m$ so $\left\|x_{m}-y_{m}\right\|$ is independent of $m$ for large $m$ and $\mu>0$ since $x_{m} \neq y_{m}$.

Case (ii): $\left(k_{m}^{i}\right)$ and $\left(p_{m}^{s}\right)$ are bounded, $1 \leq i \leq j_{1}, 0 \leq s \leq s_{1}$, and unbounded $j_{1}<i \leq j, s_{1}<s \leq r$. Then

$$
\begin{aligned}
\left\|x_{m}-y_{m}\right\|= & \left\|\sum_{i=1}^{j_{1}} \tau_{k_{m}^{i}} v_{i}-\sum_{i=1}^{s_{1}} \tau_{p_{m}^{i}} w_{i}\right\| \\
& +\left\|\sum_{i=j_{1}+1}^{j} \tau_{k_{m}^{s}} v_{i}-\sum_{i=s_{1}+1}^{s} \tau_{p_{m}^{i}} w_{i}\right\|+\varepsilon_{m},
\end{aligned}
$$

where $\varepsilon_{m} \rightarrow 0$ as $m \rightarrow \infty$. As in Case (i), the first term on the right in (1.57) can be assumed to be independent of $m$ for large $m$. Therefore either it is positive, in which case $\mu>0$, or it equals 0 and

$$
\mu=\lim _{m \rightarrow \infty}\left\|\sum_{i=j_{1}+1}^{j} \tau_{k_{m}^{i}} v_{i}-\sum_{i=s_{1}+1}^{s} \tau_{p_{m}^{i}} w_{i}\right\| .
$$

Thus we have returned to a situation like (1.56) but with at least one fewer term except that now one sum may be vacuous, i.e., $j=j_{1}$ or $s=s_{1}$. Suppose that $j=j_{1}$. Note that

$$
\left\|\sum_{s_{1}+1}^{s} \tau_{p_{m}^{i}} w_{i}\right\|=\left\|\sum_{i=s_{1}+1}^{s} \tau_{p_{m}^{i}-p_{m}^{s_{1}+1}} w_{i}\right\| .
$$

If $\left(p_{m}^{i}-p_{m}^{s_{1}+1}\right)$ is bounded, $s_{1}+1<i \leq s$, then we are in the setting of Case (i) again and can assume $p_{m}^{i}-p_{m}^{s_{1}+1}$ is independent of $m$ for large $m$. Hence if (1.59) is positive, then $\mu>0$, while if (1.59) is zero, then $x_{m}=y_{m}$ for large $m$ which is impossible.

The remaining cases are treated by repeating the above arguments. Thus in a finite number of steps we conclude $\mu>0$.

\section{A DEFORMATION THEOREM}

This section contains a version of a standard deformation theorem for $I^{b}$, $b<c+\alpha$, assuming that $\left(\mathrm{V}_{1}\right)-\left(\mathrm{V}_{3}\right)$ are satisfied and

(*) There exists an $\alpha>0$ such that $I^{c+\alpha}$ contains finitely many normalized critical points. 
Without loss of generality, we can assume

$$
\alpha<\underline{c} / 3 \text {. }
$$

The Deformation Theorem will be applied to construct an approximating "minimaxing" curve for $c$ in (0.4) having certain special properties (Proposition 2.22). Moreover the ingredients in its proof are employed in our main existence results. As a simple application of the Deformation Theorem, it will also be shown that $c$ is a critical value for $I$.

To begin we recall that one of the key roles (PS) plays in the proof of the "standard" Deformation Theorem (see, e.g., [6]) is that it provides a $\delta>0$ such that $\left\|I^{\prime}(x)\right\| \geq \delta$ for all $x \in I_{b-\varepsilon}^{b+\varepsilon}$ for some $\varepsilon>0$ if $\mathscr{K}(b) \equiv \mathscr{K}_{b}^{b}=\varnothing$ and an appropriately modified statement if $\mathscr{K}(b) \neq \varnothing$. Since (PS) fails in our setting, there is no such $\delta$ but we have the following result. Here $N_{r}(A)=$ $\{x \in E \mid\|x-A\|<r\}$.

Proposition 2.2. Let $\left(\mathrm{V}_{1}\right)-\left(\mathrm{V}_{3}\right)$ and $(*)$ hold. If $\mathscr{F}$ denotes the set of normalized critical points in $I^{c+\alpha}$ and $r<\frac{1}{3} \mu\left(\mathscr{T}_{\bar{l}}(\mathscr{F})\right)$, where $\bar{l}=[(c+\alpha) / \underline{c}]$, then there is a $\delta>0$ such that

$$
\left\|I^{\prime}(x)\right\| \geq \delta \text { for } x \in I_{c-\alpha}^{c+\alpha} \backslash N_{r / 8}(\mathscr{T}(\mathscr{F})) .
$$

Proof. If not, there is a sequence $\left(x_{m}\right) \subset I_{c-\alpha}^{c+\alpha} \backslash N_{r / 8}(\mathscr{T}(\mathscr{F}))$ such that $I^{\prime}\left(x_{m}\right) \rightarrow$ 0 and $I\left(x_{m}\right) \rightarrow \gamma \in[c-\alpha, c+\alpha]$. By Proposition 1.24, along a subsequence, $x_{m} \rightarrow \mathscr{T}(\mathscr{F})$, contrary to $x_{m} \notin N_{r / 8}(\mathscr{T}(\mathscr{F}))$.

Now we can prove the Deformation Theorem. Let $\mathscr{K}_{\beta}^{\gamma}=I_{\beta}^{\gamma} \cap \mathscr{K}$.

Proposition 2.3 (Deformation Theorem). Let $V$ satisfy $\left(\mathrm{V}_{1}\right)-\left(\mathrm{V}_{3}\right)$ and let $(*)$ hold. If $b \in(0, c+\alpha)$, then for any $\bar{\varepsilon} \in(0, \alpha]$ and $r<\frac{1}{3} \mu(\mathscr{T}(\mathscr{F}))$, there exist $\varepsilon \in(0, \bar{\varepsilon}), \eta \in C([0,1] \times E, E)$, and $\sigma \in C\left(I^{b+\varepsilon},[0,1]\right)$ such that:

$1^{\circ} . \eta(0, x)=x$ for all $x \in E$,

$2^{\circ} . \eta(s, x)=x$ if $x \notin I_{b-\bar{\varepsilon}}^{b+\bar{\varepsilon}}$,

$3^{\circ}$. I $(\eta(s, x))$ is nonincreasing in $s$,

$4^{\circ} \cdot \eta\left(1, I^{b+\varepsilon} \backslash N_{r}\left(\mathscr{K}_{b-\bar{\varepsilon}}^{b+\bar{\varepsilon}}\right)\right) \subset I^{b-\varepsilon}$,

$5^{\circ} . \sigma(x)=0$ if $x \in I^{b-\varepsilon} \backslash N_{r}\left(\mathscr{K}_{b-\bar{\varepsilon}}^{b+\bar{\varepsilon}}\right)$ and $I(\eta(\sigma(x), x))=b-\varepsilon$ for all $x \in I_{b-\varepsilon}^{b+\varepsilon} \backslash N_{r}\left(\mathscr{K}_{b-\bar{\varepsilon}}^{b+\bar{\varepsilon}}\right)$,

$6^{\circ}$. $\|\eta(\sigma(x), x)-x\| \leq r$ for all $x \in E$,

$7^{\circ} . \eta\left(s, \tau_{k} x\right)=\tau_{k} \eta(s, x)$ for all $k \in \mathbf{Z}, s \in[0,1]$, and $x \in E$.

Proof. Let

$$
\varphi(x)=\frac{\left\|x-N_{r / 8}\left(\mathscr{K}_{b-\bar{\varepsilon}}^{b+\bar{\varepsilon}}\right)\right\|}{\left\|x-N_{r / 8}\left(\mathscr{K}_{b-\bar{\varepsilon}}^{b+\bar{\varepsilon}}\right)\right\|+\left\|x-E \backslash N_{r / 4}\left(\mathscr{K}_{b-\bar{\varepsilon}}^{b+\bar{\varepsilon}}\right)\right\|} .
$$

Let $\hat{\varepsilon}<\bar{\varepsilon}$ and set

$$
f(x)=\frac{\left\|x-\left(I^{b-\hat{\varepsilon}} \cup I_{b+\hat{\varepsilon}}\right)\right\|}{\left\|x-\left(I^{b-\hat{\varepsilon}} \cup I_{b+\hat{\varepsilon}}\right)\right\|+\left\|x-I_{b-\varepsilon}^{b+\varepsilon}\right\|},
$$


where $\varepsilon<\hat{\varepsilon}$ is free for now. Let $\mathscr{V}$ be a locally Lipschitz continuous function on $E \backslash \mathscr{K}$ such that

$$
\begin{array}{ll}
\text { (i) } & \|\mathscr{V}(x)\| \leq \frac{4 \hat{\varepsilon}}{\left\|I^{\prime}(x)\right\|}, \\
\text { (ii) } I^{\prime}(x) \mathscr{V}(x) \geq 2 \hat{\varepsilon}, \\
\text { (iii) } \mathscr{V}\left(\tau_{k} x\right)=\mathscr{V}(x) \text { for all } k \in \mathbf{Z}, \quad x \in E \backslash \mathscr{K},
\end{array}
$$

i.e., $\mathscr{V}$ is an appropriately scaled pseudogradient vector field. The existence of such a $\mathscr{V}$ follows from [2] or [6].

The function $\eta$ will be determined as the solution of an ordinary differential equation corresponding to $\mathscr{V}$. Set $W(x)=-f(x) \varphi(x) \mathscr{V}(x)$ and let $\eta(\cdot, x)$ be the local solution of

$$
\frac{d \eta}{d s}=W(\eta), \quad \eta(s, x)=x .
$$

It is clear that $\eta$ satisfies $1^{\circ}, 2^{\circ}$, and, because of $(2.6)(\mathrm{iii}), 7^{\circ}$. Since

$$
\frac{d}{d s} I(\eta(x, s))=-f(\eta(x, s)) \phi(\eta(s, x)) I^{\prime}(\eta(s, x)) \mathscr{V}(\eta(s, x)) \leq 0,
$$

$3^{\circ}$ holds. Before verifying the remaining assertions of the theorem, we will show that $\eta(s, x)$ exists for all $s>0$. This is obvious for $x \in I^{b-\hat{\varepsilon}} \cup I_{b+\hat{\varepsilon}} \cup$ $\bar{N}_{r / 8}\left(\mathscr{K}_{b-\bar{\varepsilon}}^{b+\bar{\varepsilon}}\right) \equiv Y$. Thus suppose $x \notin Y$ and consider $\eta(s, x)$. If $\eta(s, x)$ exists only up to $\bar{s}(x)$, let $s_{m} \nearrow \bar{s}$ and $u_{m}=\eta\left(s_{m}, x\right)$. Then $I\left(u_{m}\right) \rightarrow$ $\bar{b} \in[b-\hat{\varepsilon}, b+\hat{\varepsilon}], I^{\prime}\left(u_{m}\right) \rightarrow 0$, and $u_{m} \rightarrow \mathscr{T}(\mathscr{F})$ as in Proposition 2.2. By Proposition 1.55, $\mathscr{T}(\mathscr{F})$ consists of isolated points. Therefore either (i) $u_{m} \in B_{r / 2}(u)$ for some $u \in \mathscr{T}(\mathscr{F})$ for all large $m$, or (ii) $u_{m}$ enters infinitely many such $B_{r / 2}$. If (i), then $u_{m} \rightarrow u$ and therefore $I^{\prime}(u)=0$ contrary to $u_{m} \notin N_{r / 8}\left(\mathscr{K}_{b-\bar{\varepsilon}}^{b+\bar{\varepsilon}}\right)$. If (ii), then whenever $\eta(s, x)$ travels from $B_{r / 2}\left(y_{i}\right)$ to $B_{r / 2}\left(y_{j}\right)$, where $y_{i}, y_{j} \in \mathscr{T}(\mathscr{F})$ and $i \neq j$, we have for some $\hat{s}_{i}<\hat{s}_{j}<\bar{s}(x)$ :

$$
\begin{aligned}
r & <\left\|\eta\left(\hat{s}_{j}, x\right)-\eta\left(\hat{s}_{i}, x\right)\right\|=\left\|\int_{\hat{s}_{i}}^{\hat{s}_{j}} \frac{d \eta}{d s} d s\right\| \\
& =\left\|\int_{\hat{s}_{i}}^{\hat{s}_{j}} f \varphi V d s\right\| \leq \frac{4 \hat{\varepsilon}}{\delta} \int_{\hat{s}_{i}}^{\hat{s}_{j}} f \varphi d s
\end{aligned}
$$

via (2.6)(i) and Proposition 2.2. On the other hand, by (2.6)(ii), if $\eta$ meets $k+1$ such balls in the interval $(s, \hat{s})$, then

$$
\begin{aligned}
2 \hat{\varepsilon} & \geq I(\eta(s, x))-I(\eta(\hat{s}, x))=\int_{\hat{s}}^{s} \frac{d I}{d s} d s \\
& \geq \sum_{l=1}^{k} \int_{\hat{s}_{i(l)}}^{\hat{s}_{j(l)}} f \varphi I^{\prime} \mathscr{V} d s \geq 2 \hat{\varepsilon} \sum_{l=1}^{k} \int_{\hat{s}_{i(l)}}^{\hat{s}_{j(l)}} f \varphi d s .
\end{aligned}
$$

Combining (2.9) $-(2.10)$ shows

$$
4 \hat{\varepsilon} \geq k r \delta
$$


and since $k$ is arbitrary, it follows that $\eta(s, x)$ exists for all $s>0$.

Next we show there is a $\sigma \in C\left(I^{b+\varepsilon},[0,1]\right)$ such that

$$
I(\eta(\sigma(x), x)) \leq b-\varepsilon \quad \text { for } x \in I^{b+\varepsilon} \backslash N_{r}\left(\mathscr{K}_{b-\bar{\varepsilon}}^{b+\bar{\varepsilon}}\right) .
$$

Then $3^{\circ}$ and (2.12) imply $4^{\circ}$. An argument like (2.9)-(2.11) will be employed. Two cases will be considered to prove (2.12).

Case 1: $x \in I_{b-\varepsilon}^{b+\varepsilon} \backslash N_{r / 4}\left(\mathscr{T}_{\bar{l}}(\mathscr{F})\right)$. Then the orbit $\eta(s, x)$ either (i) intersects $\partial N_{r / 8}(\mathscr{T}(\mathscr{F}))$ or (ii) intersects $\partial I^{b-\varepsilon}$. If (i), there is a time interval of length $\theta(x)$ in which the orbit remains in $N_{r / 4}(\mathscr{T}(\mathscr{F}))$ and runs from $\partial N_{r / 4}(\mathscr{T}(\mathscr{F}))$ to $\partial N_{r / 8}(\mathscr{T}(\mathscr{F}))$. Then, as in (2.9),

$$
\begin{aligned}
\frac{r}{8} & =\|\eta(s+\theta(x), x)-\eta(s, x)\|=\left\|\int_{s}^{s+\theta(x)} \frac{d \eta}{d s} d s\right\| \\
& \leq \int_{s}^{s+\theta(x)}\|\mathscr{V}(\eta(s, x))\| d s \leq \frac{4 \hat{\varepsilon}}{\delta} \theta(x)
\end{aligned}
$$

while (observing that $f$ and $\varphi$ equal 1 in the region under consideration), as in $(2.10)$,

$$
2 \varepsilon \geq I(x)-I(\eta(s+\theta(x), x)) \geq \int_{s+\theta(x)}^{0} \frac{d I}{d s} d s \geq 2 \hat{\varepsilon} \theta(x) .
$$

Therefore

$$
\theta(x) \leq \frac{\varepsilon}{\hat{\varepsilon}}<1
$$

and

$$
\frac{r}{8} \leq \frac{4 \varepsilon}{\delta}
$$

Choosing

$$
\varepsilon<\frac{r \delta}{40},
$$

we see that (2.16) is not possible. Therefore (ii) occurs and $\eta(s, x)$ intersects $\partial I^{b-\varepsilon}$. If $\omega(x)$ is the amount of time it takes $\eta$ to reach $\partial I^{b-\varepsilon}$, then (2.14)(2.15) show $\omega(x)<1$. Set $\sigma(x)=\omega(x)$. Thus we have $5^{\circ}$ for Case 1 . Note also that

Hence

$$
\|\eta(\sigma(x), x)-x\| \leq \frac{4 \hat{\varepsilon}}{\delta} \sigma(x) \leq \frac{4 \varepsilon}{\delta} .
$$

so $6^{\circ}$ holds for Case 1 .

Case 2: $x \in\left(I_{b-\varepsilon}^{b+\varepsilon} \cap N_{r / 4}\left(\mathscr{T}_{\bar{l}}(\mathscr{F})\right)\right) \backslash N_{r}\left(\mathscr{K}_{b-\bar{\varepsilon}}^{b+\bar{\varepsilon}}\right)$. Then $x \in B_{r / 4}(u)$ for some $u \in \mathscr{T}_{\bar{l}}(\mathscr{F}) \backslash \mathscr{K}_{b-\bar{\varepsilon}}^{b+\bar{\varepsilon}}$. Either (i) $\eta(s, x)$ remains in $B_{r / 2}(u)$ for $s \in[0,1]$ or (ii) 
$\eta(s, x)$ reaches $\partial B_{r / 2}(u)$. If (ii), the estimates of $(2.13)-(2.17)$ with $r / 8$ replaced by $r / 4$ show $\eta(s, x)$ reaches $\partial I^{b-\varepsilon}$ before it reaches $\partial B_{r / 2}(u)$. Hence (i) holds. By the argument of $(2.14)-(2.15), \eta(s, x)$ reaches $\partial I^{b-\varepsilon}$ within time 1. Also since $\eta(s, x) \in B_{r / 2}(u)$ for all $s \in[0,1]$, letting $\sigma(x)$ be the value of $s$ at which $\eta$ reaches $\partial I^{b-\varepsilon}, 6^{\circ}$ holds for this case.

To complete the proof of $5^{\circ}-6^{\circ}$, we define $\sigma(x)=0$ for $x \in I^{b-\varepsilon} \backslash N_{r}\left(\mathscr{K}_{b-\bar{\varepsilon}}^{b+\bar{\varepsilon}}\right)$. Finally, for $x \in I^{b+\varepsilon} \cap N_{r}\left(\mathscr{K}_{b-\bar{\varepsilon}}^{b+\bar{\varepsilon}}\right)$, we take

$$
\sigma(x)=\min \left(1, \text { time at which } \eta \in \partial I^{b-\varepsilon}\right)
$$

and the proof is complete.

As a simple application of Proposition 2.3, we have

Theorem 2.19. If $V$ satisfies $\left(\mathrm{V}_{1}\right)-\left(\mathrm{V}_{3}\right)$ and $(*)$ holds, $c$ is a critical value of I.

Proof. If not, by $(*)$, for $\bar{\varepsilon}$ sufficiently small, $\mathscr{K}_{c-\bar{\varepsilon}}^{c+\bar{\varepsilon}}=\varnothing$. With this choice of $\bar{\varepsilon}$ and $r<\frac{1}{3} \mu(\mathscr{T}(\mathscr{F}))$, let $\varepsilon$ be as given by Proposition 2.3 and $g \in \Gamma$ such that

$$
\max _{\theta \in[0,1]} I(g(\theta)) \leq c+\varepsilon .
$$

Then by $4^{\circ}$ of Proposition 2.3,

$$
\max _{\theta \in[0,1]} I(\eta(1, g(\theta))) \leq c-\varepsilon .
$$

But by $2^{\circ}$ of Proposition $2.3, \eta(1, g) \in \Gamma$ so $(2.21)$ is contrary to the definition of $c$.

Theorem 2.19 is not needed in the sequel but the following result is important in the proof of our main existence result. Note that if $(*)$ is satisfied, then

$$
0<\alpha_{1} \equiv \sup \left\{\beta<\alpha \mid \mathscr{K}_{c-\beta}^{c+\beta}=\mathscr{K}(c)\right\} .
$$

Proposition 2.22. Let $V$ satisfy $\left(\mathrm{V}_{1}\right)-\left(\mathrm{V}_{3}\right)$ and $(*)$. Then there exists a finite set $A \subset \mathscr{K}(c)$ such that for all $\bar{\varepsilon}_{1} \leq \alpha_{1} / 2, r_{1} \leq \frac{1}{12} \mu(\mathscr{T}(\mathscr{F}))$, and $p \in \mathbf{N}$ there is an $\varepsilon_{1} \in\left(0, \bar{\varepsilon}_{1}\right)$ and $g_{1} \in \Gamma$ such that

$$
\begin{aligned}
& 1^{\circ} \cdot \max _{\theta \in[0,1]} I\left(g_{1}(\theta)\right) \leq c+\varepsilon_{1} / p \text { and } \\
& 2^{\circ} \cdot I\left(g_{1}(\theta)\right)>c-\varepsilon_{1} \text { implies } g_{1}(\theta) \in N_{r_{1}}(A) .
\end{aligned}
$$

Proof. Setting $b=c, r=r_{0} / 2 \equiv \frac{1}{24} \mu(\mathscr{T}(\mathscr{F})), \bar{\varepsilon}=\bar{\varepsilon}_{0} \equiv \alpha_{1} / 2$, we invoke Proposition 2.3 obtaining $\varepsilon_{0}=\varepsilon$, and $\eta_{0}, \sigma_{0}$ as in the statement of the proposition. Choose $g \in \Gamma$ such that

$$
\max _{\theta \in[0,1]} I(g(\theta)) \leq c+\frac{\varepsilon_{0}}{p} .
$$

Therefore if $g_{0}=\eta_{0}\left(\sigma_{0}(g), g\right)$, by $3^{\circ}$ of Proposition 2.3,

$$
\max _{\theta \in[0,1]} I\left(\eta_{0}\left(\sigma_{0}(g), g\right)\right) \leq c+\frac{\varepsilon_{0}}{p}
$$


so $1^{\circ}$ above holds with $g_{1} \rightarrow g_{0}, \varepsilon_{1} \rightarrow \varepsilon_{0}$. Moreover, by $5^{\circ}$ of Proposition 2.3 , if $I\left(g_{0}(\theta)\right)>c-\varepsilon_{0}$, then $g(\theta) \in N_{r}(\mathscr{K}(c))$. By $6^{\circ}$ of Proposition 2.3,

$$
\left\|g_{0}(\theta)-g(\theta)\right\| \leq r .
$$

Hence $I\left(g_{0}(\theta)\right)>c-\varepsilon_{0}$ implies $g_{0}(\theta) \in N_{r_{0}}(\mathscr{K}(c))$.

Next we claim there is a set $A$ so that $2^{\circ}$ holds with $g_{1} \rightarrow g_{0}, \varepsilon_{1} \rightarrow \varepsilon_{0}$, and $r_{1} \rightarrow r_{0}$. If not, there exists a sequence of distinct $v_{m} \in \mathscr{K}(c)$ and $\theta_{m} \in[0,1]$ such that

$$
\left\|g_{0}\left(\theta_{m}\right)-v_{m}\right\| \leq r_{0} .
$$

By compactness, there is a $\bar{\theta} \in[0,1]$ such that $\theta_{m} \rightarrow \bar{\theta}$ along a subsequence. For such $m$,

$$
\begin{aligned}
\left\|v_{m_{i}}-v_{m_{j}}\right\| \leq & \left\|v_{m_{i}}-g_{0}\left(\theta_{m_{i}}\right)\right\|+\left\|g_{0}\left(\theta_{m_{i}}\right)-g_{0}\left(\theta_{m_{j}}\right)\right\| \\
& +\left\|g_{0}\left(\theta_{m_{j}}\right)-v_{m_{j}}\right\| \\
\leq & 2 r_{0}+\left\|g_{0}\left(\theta_{m_{i}}\right)-g_{0}\left(\theta_{m_{j}}\right)\right\| \rightarrow 2 r_{0} \leq \frac{1}{6} \mu\left(\mathscr{T}_{\bar{l}}(\mathscr{F})\right),
\end{aligned}
$$

contrary to Proposition 1.55. Therefore there is a finite set $A$ such that $2^{\circ}$ holds for this particular choice of $\bar{\varepsilon}_{1}, r_{1}$, and $p$.

Now take any $\bar{\varepsilon}_{1} \leq \bar{\varepsilon}_{0}, r_{1} \leq r_{0}$, and $p \in \mathbf{N}$.

Setting $b=c, r=r_{1} / 2$, and $\bar{\varepsilon}=\bar{\varepsilon}_{1}$ we invoke again Proposition 2.3 obtaining $\varepsilon_{1}=\varepsilon$ and $\eta_{1}, \sigma_{1}$ as in the statement of the proposition.

Now choose $\rho$ so that

$$
\max _{x \in N_{\rho}\left(\mathscr{H}_{b-\varepsilon_{0}}^{b+\bar{\varepsilon}_{0}}\right)} I(x)<c+\frac{\varepsilon_{1}}{p}
$$

and define

$$
\hat{\varphi}(x)=\frac{\left\|x-N_{\rho / 8}\left(\mathscr{K}_{b-\bar{\varepsilon}_{0}}^{b+\bar{\varepsilon}_{0}}\right)\right\|}{\left\|x-N_{\rho / 8}\left(\mathscr{K}_{b-\bar{\varepsilon}_{0}}^{b+\bar{\varepsilon}_{0}}\right)\right\|+\left\|x-E \backslash N_{\rho / 4}\left(\mathscr{K}_{b-\bar{\varepsilon}_{0}}^{b+\bar{\varepsilon}_{0}}\right)\right\|} .
$$

Setting $\hat{\varepsilon}=\max \left\{\bar{\varepsilon}_{1}, \varepsilon_{0}\right\} \leq \bar{\varepsilon}_{0}$, we define

$$
\hat{f}(x)=\frac{\left\|x-\left(I^{b-\bar{\varepsilon}_{0}} \cup I_{b+\bar{\varepsilon}_{0}}\right)\right\|}{\left\|x-\left(I^{b-\bar{\varepsilon}_{0}} \cup I_{b+\bar{\varepsilon}_{0}}\right)\right\|+\left\|x-I_{b-\hat{\varepsilon}}^{b+\hat{\varepsilon}}\right\|}
$$

and we let $V$ satisfy (2.6) with the $\bar{\varepsilon}$ defined here. We now denote by $\xi(s, x)$ the solution of

$$
\frac{d \xi}{d s}=-\hat{f}(\xi) \hat{\varphi}(\xi) V(\xi), \quad \xi(0, x)=x .
$$

As in Proposition $2.3 \xi(s, x)$ is defined for all $s \geq 0$.

Let $\tilde{g}(\theta)=\xi\left(1, g_{0}(\theta)\right)$. If, for some $\bar{\theta} \in[0,1]$,

$$
I(\tilde{g}(\bar{\theta}))>c+\frac{\varepsilon_{1}}{p}
$$


then by $3^{\circ}$ of Proposition 2.3 and (2.23),

$$
c+\frac{\varepsilon_{1}}{p}<I\left(\xi\left(s, g_{0}(\bar{\theta})\right)\right) \leq c+\varepsilon_{0} \leq c+\hat{\varepsilon} \quad \forall s \in[0,1] .
$$

Hence (2.28)-(2.29) show

$$
\hat{\varphi}\left(\xi\left(s, g_{0}(\bar{\theta})\right)\right) \hat{f}\left(\xi\left(s, g_{0}(\bar{\theta})\right)\right)=1 \quad \forall s \in[0,1] .
$$

As in Proposition 2.3 one deduces that

$$
I\left(g_{0}(\bar{\theta})\right)-I(\tilde{g}(\bar{\theta})) \geq 2 \hat{\varepsilon},
$$

contrary to (2.29). Therefore

$$
I(\tilde{g}(\theta)) \leq c+\frac{\varepsilon_{1}}{p} \quad \forall \theta \in[0,1] .
$$

We finally define

$$
g_{1}(\theta)=\eta_{1}\left(\sigma_{1}(\tilde{g}(\theta)), \tilde{g}(\theta)\right) .
$$

Then by (2.30) and $3^{\circ}$ and $5^{\circ}$ of Proposition 2.3,

$$
\max _{\theta \in[0,1]} I\left(g_{1}(\theta)\right) \leq \max _{\theta \in[0,1]} I(\tilde{g}(\theta)) \leq c+\frac{\varepsilon_{1}}{p}
$$

and

$$
I\left(g_{1}(\theta)\right)>c-\varepsilon_{1} \quad \text { implies } \quad g_{1}(\theta) \in B_{r_{1}}(\mathscr{K}) .
$$

To show that, actually,

$$
I\left(g_{1}(\theta)\right)>c-\varepsilon_{1} \quad \text { implies } \quad g_{1}(\theta) \in B_{r_{1}}(A)
$$

suppose

$$
I\left(g_{1}(\bar{\theta})\right)>c-\varepsilon_{1}
$$

and

$$
g_{1}(\bar{\theta}) \in N_{r_{1}}(\mathscr{K}) \backslash N_{r_{1}}(A)
$$

Since

$$
I\left(g_{0}(\bar{\theta})\right) \geq I\left(g_{1}(\bar{\theta})\right)>c-\varepsilon_{1} \geq c-\varepsilon_{0},
$$

we have that $g_{0}(\bar{\theta}) \in N_{r_{0}}(A)$. Consider the trajectory $\xi\left(s, g_{0}(\bar{\theta})\right), 0 \leq s \leq 1$, followed by $\eta_{1}\left(s_{1}, \tilde{g}(\bar{\theta})\right), 0 \leq s_{1} \leq \sigma(\tilde{g}(\bar{\theta}))$. It takes $g_{0}(\bar{\theta}) \in N_{r_{0}}(A)$ to $g_{1}(\bar{\theta}) \in N_{r_{1}}\left(\mathscr{K} \backslash N_{r_{1}}(A)\right)$. Since $r_{1} \leq r_{0}$ and $\left\|N_{r_{0}}(A)-N_{r_{1}}(\mathscr{K}) \backslash N_{r_{1}}(A)\right\| \geq 10 r_{0}$ via the choice of $r_{0}, g_{0}(\bar{\theta})$ moves a distance $\geq 10 r_{0}$ through a region in which $\left\|I^{\prime}\right\| \geq \delta_{0}=\delta_{0}\left(r_{0}\right)$, the value of $\delta$ given by Proposition 2.2. But the arguments of (2.13)-(2.17) show this is impossible. Hence (2.31) and (2.33) hold and the proof is complete.

\section{SOME EXISTENCE RESULTS}

In this section we will establish the existence of infinitely many solutions of (HS) in $\mathscr{K}_{k c-\alpha}^{k c+\alpha} / \mathbf{Z}$ for all $k \in \mathbf{N} \backslash\{1\}$. In fact we will prove the existence 
of solutions of a certain form. The first step in this process is to define a class of sets. For $k \in \mathbf{N} \backslash\{1\}$ and $\theta=\left(\theta_{1}, \ldots, \theta_{k}\right) \in[0,1]^{k}$, let $0_{i}=$ $\left(\theta_{1}, \ldots, \theta_{i-1}, 0, \theta_{i+1}, \ldots, \theta_{k}\right)$ and $1_{i}=\left(\theta_{1}, \ldots, \theta_{i-1}, 1, \theta_{i+1}, \ldots, \theta_{k}\right)$. For $q \in E, \operatorname{supp} q$ denotes the support of $q$ (as a mapping of $\mathbf{R}$ to $\mathbf{R}^{n}$ ). Let

$$
\Gamma_{k}=\left\{G=g_{1}+\cdots+g_{k} \mid \text { satisfying }\left(\mathbf{g}_{1}\right)-\left(\mathbf{g}_{3}\right)\right\}
$$

where

$\left(\mathrm{g}_{1}\right) \quad g_{i} \in C\left([0,1]^{k}, E\right), 1 \leq i \leq k ;$

$\left(\mathrm{g}_{2}\right) \quad g_{i}\left(0_{i}\right)=0, g_{i}\left(1_{i}\right) \in I^{0} \backslash\{0\}, 1 \leq i \leq k$;

$\left(\mathrm{g}_{3}\right)$ there exist $p_{1}<p_{2}<\cdots<p_{k-1}$ independent of $\theta$ such that

$$
\operatorname{supp} g_{1}(\theta) \subset\left(-\infty, p_{1}\right), \operatorname{supp} g_{2} \subset\left(p_{1}, p_{2}\right), \ldots, \operatorname{supp} g_{k}(\theta) \subset\left(p_{k-1}, \infty\right) \text {. }
$$

Remark 3.1. Note that

$$
\Gamma_{k} \supset\left\{\sum_{i=1}^{k} g_{i}\left(\theta_{i}\right) \mid g_{i} \in \Gamma, 1 \leq i \leq n \text { and }\left(\mathrm{g}_{3}\right) \text { holds }\right\} .
$$

Set

$$
b_{k}=\inf _{G \in \Gamma_{k}} \max _{\theta \in[0,1]^{k}} I(G(\theta)) .
$$

Observe that if $G \in \Gamma_{k}$, then by $\left(\mathrm{g}_{3}\right)$

$$
I(G)=\sum_{i=1}^{k} I\left(g_{i}\right)
$$

Proposition 3.4. Let $g_{i}$ satisfy $\left(\mathrm{g}_{1}\right)-\left(\mathrm{g}_{2}\right), 1 \leq i \leq k$. Then there exists $a$ $\bar{\theta} \in[0,1]^{k}$ such that $I\left(g_{i}(\bar{\theta})\right) \geq c, 1 \leq i \leq k$.

Proof. Consider $I\left(g_{1}(\theta)\right)$. Since $g_{1}\left(0_{1}\right)=0$ and $g_{1}\left(1_{1}\right) \in I^{0} \backslash\{0\}$, every curve joining $\{0\} \times[0,1]^{k-1}$ to $\{1\} \times[0,1]^{k-1}$ (in $[0,1]^{k}$ ) lies in $\Gamma$ and therefore intersects $I^{-1}(c)$ via the definition of $c$. It follows that $\left(I\left(g_{1}\right)\right)^{-1}(c)$ separates $\{0\} \times[0,1]^{k-1}$ and $\{1\} \times[0,1]^{k-1}$ (in $\left.[0,1]^{k}\right)$. Let $\varepsilon>0$. Then for $\delta_{1}$ sufficiently small, $I\left(g_{1}\right) \geq c-\varepsilon$ in a uniform $\delta_{1}$ neighborhood of $\left(I\left(g_{1}\right)\right)^{-1}(c)$. Since $[0,1]^{k}$ is compact this neighborhood contains only finitely many components. Hence at least one component separates $\{0\} \times[0,1]^{k-1}$ and $\{1\} \times[0,1]^{k-1}$. Denote this component by $\mathscr{D}_{1}$. Note that $\mathscr{D}_{1}$ is an arcwise connected set joining $\theta_{2}=0$ to $\theta_{2}=1$ and, as earlier, $I\left(g_{2}\right)=c$ somewhere on each curve in $\mathscr{D}_{1}$ joining $\theta_{2}=0$ to $\theta_{2}=1$. Therefore $\left(I\left(g_{2}\right)\right)^{-1}(c)$ separates $\mathscr{D}_{1} \cap\left\{\theta_{2}=0\right\}$ and $\mathscr{D}_{1} \cap\left\{\theta_{2}=1\right\}$ in $\mathscr{D}_{1}$. For $\delta_{2}$ sufficiently small, $I\left(g_{2}\right) \geq c-\varepsilon$ in a uniform $\delta_{2}$ neighborhood of $\left(I\left(g_{2}\right)\right)^{-1}(c) \cap \mathscr{D}_{1}$ and this neighborhood contains finitely many components. Hence at least one of them, which we denote by $\mathscr{D}_{2}$, separates $\mathscr{D}_{1} \cap\left\{\theta_{2}=0\right\}$ and $\mathscr{D}_{1} \cap\left\{\theta_{2}=1\right\}$. Continuing this process, after $k-1$ steps, we construct a compact connected set $\mathscr{D}_{k-1} \subset \cdots \subset \mathscr{D}_{2} \subset \mathscr{D}_{1}$, 
such that $I\left(g_{i}\right) \geq c-\varepsilon$ in $\mathscr{D}_{k-1}, 1 \leq i \leq k-1$. Since $\mathscr{D}_{k-1}$ connects $\theta_{k}=0$ to $\theta_{k}=1$, there exists a $\bar{\theta}_{\varepsilon} \in \mathscr{D}_{k-1}$ such that $I\left(g_{k}\left(\bar{\theta}_{\varepsilon}\right)\right)=c$. Letting $\varepsilon \rightarrow 0$, there is a subsequence $\bar{\theta}_{\varepsilon} \rightarrow \bar{\theta}$ such that $I\left(g_{i}(\bar{\theta})\right) \geq c, 1 \leq i \leq k$, and the proof is complete.

Proposition 3.5. $b_{k}=k c$.

Proof. Proposition 3.4 and (3.3) show

$$
\max _{\theta \in[0,1]^{k}} I(G(\theta)) \geq k c
$$

for each $G \in \Gamma_{k}$.Hence $b_{k} \geq k c$. On the other hand, let $\varepsilon>0$. By the definition of $c$, there is a $g \in \Gamma$ such that

$$
\max _{s \in[0,1]} I(g(s)) \leq c+\frac{\varepsilon}{2 k}
$$

By appropriately truncating $g(\theta)(t)$ for large $|t|$ (uniformly in $\theta$ ) we obtain $R>0$ and $\hat{g} \in \Gamma$ such that $\operatorname{supp} \hat{g}(s) \subset[-R, R]$ and

$$
\max _{s \in[0,1]} I(\hat{g}(s)) \leq c+\frac{\varepsilon}{k} .
$$

Thus if $j T>2 R$, then $G(\theta)=\sum_{i=1}^{k} \tau_{(i-1) j} \hat{g}\left(\theta_{i}\right) \in \Gamma_{k}$ and, by (3.7),

$$
\max _{\theta \in[0,1]^{n}} I(G(\theta)) \leq k c+\varepsilon .
$$

Since $\varepsilon$ is arbitrary, it follows that $b_{k} \leq k c$ and the result is proved.

Remark. A set related to $\Gamma_{2}$ and an analogue of Proposition 3.5 for $b_{2}$ were proved in his setting by Séré [3].

To prove that $\mathscr{K}_{k c-\alpha}^{k c+\alpha} / \mathbf{Z}$ is infinite, the idea is to argue indirectly and show that, if not, there is an $H \in \Gamma_{k}$ such that $I(H)<k c$, contrary to Proposition 3.5. Actually this will be done in such a way as to give more information about the form of solutions. To be more precise, let $A \subset \mathscr{K}(c)$ be given by Proposition 2.22. With this choice of $A$, we will construct an associated class of sets. Let $N=\left(n_{1}, \ldots, n_{k}\right) \in \mathbf{Z}^{k}$ with $n_{i+1}-n_{i} \geq n_{0}$. Here $n_{0}$ is chosen so that

$$
\left\|\sum_{i=1}^{k} \tau_{n_{i}} v_{i}\right\| \geq k \frac{\nu}{2}
$$

(see Remark 1.19) for all choices of $v_{i} \in A$. Let

$$
\mathscr{M}(k, N, A)=\left\{\sum_{i=1}^{k} \tau_{n_{i}} v_{i} \mid v_{i} \in A\right\}
$$

and

$$
\mathscr{M}^{*}(k, N, A)=\bigcup_{l \in \mathbf{N}} \mathscr{M}(k, l N, A)
$$


Proposition 3.12. There is an $r_{k}=r_{k}(\alpha)>0$ and $n_{0}(A, \alpha)$ such that if $r \leq r_{k}$ and $z \in \bar{N}_{r}\left(\mathscr{M}^{*}(k, N, A)\right) \cap \mathscr{K}$, then $z \in \mathscr{K}_{k c-\alpha}^{k c+\alpha}$.

Proof. If $z \in \bar{N}_{r}\left(\mathscr{M}^{*}(k, N, A)\right)$, then

$$
\left\|z-\sum_{i=1}^{k} \tau_{l n_{i}} v_{i}\right\| \leq r
$$

for some $l \in \mathbf{N}$ and $v_{i} \in A, 1 \leq i \leq k$. Let $x=\sum_{i=1}^{k} \tau_{l_{i}} v_{i}$. By the Mean Value Theorem,

$$
I(z)-I(x)=I^{\prime}(y)(z-x),
$$

where $y$ lies on the "segment" joining $z$ and $x$. Therefore since we can assume $r_{k} \leq 1$,

$$
\|y\| \leq \sum_{i=1}^{k}\left\|v_{i}\right\|+1 \leq k\left(\frac{2 \mu c}{\mu-2}\right)^{1 / 2}+1
$$

via Lemma 1.21. Since $I^{\prime}$ is bounded on bounded sets,

$$
\left\|I^{\prime}(y)\right\| \leq \max _{\|w\| \leq k(2 \mu c /(\mu-2))^{1 / 2}+1}\left\|I^{\prime}(w)\right\|=M_{k} .
$$

We can choose $n_{0}=n_{0}(A, \alpha)$ so large that

$$
\left|I(x)-\sum_{i=1}^{k} I\left(v_{i}\right)\right|=|I(x)-k c|<\alpha / 2 .
$$

Choose $r_{k} \leq 1$ so that

$$
M_{k} r_{k} \leq \alpha / 2 \text {. }
$$

Combining (3.14)-(3.17) yields

$$
|I(z)-k c|<\alpha
$$

and the proposition is proved.

Remark 3.19. We can assume $r_{k} \leq r_{k-1} \leq \cdots \leq r_{1}$.

Proposition 3.20. Let $V$ satisfy $\left(\mathrm{V}_{1}\right)-\left(\mathrm{V}_{3}\right)$, and let $(*)$ hold, and let $r$ satisfy

$$
r<\min \left(\frac{1}{12} \mu\left(\tau_{\bar{l}}(\mathscr{F})\right), \nu / 2, r_{k}\right) \text {. }
$$

Then

(i) there is a $\delta_{l}=\delta_{l}(k, N, A, r)$ such that $\left\|I^{\prime}(z)\right\| \geq \delta_{l}$ for all $x \in$ $N_{r}(\mathscr{M}(k, l N, A))$, or

(ii) there is a $z \in \bar{N}_{r}(\mathscr{M}(k, l N, A))$ such that $I^{\prime}(z)=0$.

Moreover, if

$$
\mathscr{L}=\bigcup\{l \in \mathbf{N} \mid(\mathbf{i}) \text { holds for } \mathscr{M}(k, l N, A)\}
$$


and

$$
\mathscr{W}=\bigcup_{l \in \mathscr{L}} \mathscr{M}(k, l N, A)
$$

then there exists $\delta=\delta(k, N, A)$ (independent of l) such that $\left\|I^{\prime}(x)\right\| \geq \delta$ for all $x \in N_{r}(\mathscr{W}) \backslash N_{r / 8}(\mathscr{W})$.

Proof. Suppose that (i) does not hold. Then there exists a sequence $\left(x_{m}\right) \subset$ $N_{r}(\mathscr{M}(k, l N, A))$ such that $I^{\prime}\left(x_{m}\right) \rightarrow 0$. The form of $I$ implies it is bounded on bounded sets in $E$. Hence since $\mathscr{M}(k, l N, A)$ is bounded in $E, I\left(x_{m}\right)$ is bounded. Therefore by Proposition 1.24, there exist $w_{1}, \ldots, w_{p} \in \mathscr{K} \backslash\{0\}$, a subsequence of $x_{m}$, and corresponding sequences $\left(k_{m}^{i}\right) \subset \mathbf{Z}, 1 \leq i \leq p$, such that

$$
\left\|x_{m}-\sum_{i=1}^{p} \tau_{k_{m}^{i}} w_{i}\right\| \rightarrow 0
$$

as $m \rightarrow \infty$, where $\left(k_{m}^{i}\right)$ satisfies (1.27). Since $A$ is a finite set, there are $v_{1}, \ldots, v_{k} \in A$ so that, along our subsequence,

$$
\left\|x_{m}-\sum_{i=1}^{k} \tau_{\ln _{i}} v_{i}\right\| \leq r .
$$

By (3.23)-(3.24),

$$
\left\|\sum_{i=1}^{k} \tau_{\ln _{i}} v_{i}-\sum_{i=1}^{p} \tau_{k_{m}^{i}} w_{i}\right\| \leq r+\varepsilon_{m},
$$

where $\varepsilon_{m} \rightarrow 0$ as $m \rightarrow \infty$. If $\left|k_{m}^{i}\right| \rightarrow \infty$ as $m \rightarrow \infty$ for all $i, 1 \leq i \leq p$, then

$$
\frac{k \nu}{2} \leq\left\|\sum_{i=1}^{k} \tau_{l n_{i}} v_{i}\right\| \leq r,
$$

contrary to (3.9) and (3.21). Thus, by (1.27), $\left|k_{m}^{i}\right|$ is bounded along a subsequence for exactly one choice of $i$, say $i=j$. Then for large $m$, without loss of generality, $k_{m}^{j}=k^{j}$ and, by (3.25),

$$
\left\|\sum_{i=1}^{k} \tau_{l n_{i}} v_{i}-\tau_{k^{j}} w_{j}\right\| \leq r .
$$

Since $\tau_{k^{j}} w_{j} \in \mathscr{K} \backslash\{0\}$, (ii) holds.

It remains to obtain the lower bound $\delta$ for $\delta_{l}$. This is trivial if $\mathscr{L}$ is a finite set. Thus suppose $\mathscr{L}$ is not bounded. Then there is a sequence $x_{m} \in$ $N_{r}\left(\mathscr{M}\left(k, l_{m} N, A\right)\right) \backslash N_{r / 8}\left(\mathscr{M}\left(k, l_{m} N, A\right)\right)$, where $l_{m} \rightarrow \infty$ and $I^{\prime}\left(x_{m}\right) \rightarrow 0$ as $m \rightarrow \infty$. As earlier there are functions $v_{k} \in A, 1 \leq i \leq k$, and $w_{1}, \ldots, w_{p} \in$ 
$\mathscr{K} \backslash\{0\}$ such that

$$
\begin{aligned}
\frac{r}{8}-\varepsilon_{m} & \leq\left\|\sum_{i=1}^{k} \tau_{l_{m} n_{i}} v_{i}-\sum_{i=1}^{p} \tau_{k_{m}^{i}} w_{i}\right\| \\
& =\left\|\sum_{i=1}^{k} \tau_{l_{m}\left(n_{i}-n_{1}\right)} v_{i}-\sum_{i=1}^{p} \tau_{k_{m}^{i}-l_{m} n_{1}} w_{i}\right\| \leq r+\varepsilon_{m},
\end{aligned}
$$

where $\varepsilon_{m} \rightarrow 0$ as $m \rightarrow \infty$. If $\left|k_{m}^{i}-l_{m} n_{1}\right| \rightarrow \infty$ as $m \rightarrow \infty, 1 \leq i \leq p$, then (3.28) and Remark 1.19 show

$$
\nu \leq\left\|v_{1}\right\| \leq r,
$$

contrary to (3.9) and (3.21) again. If $\left|k_{m}^{i}-l_{m} n_{1}\right|$ is bounded along a subsequence for, say, $i=i_{1}$, then we can assume $k_{m}^{i_{1}}-l_{m} n_{1}=k^{i_{1}}$ for $m$ large and, as earlier,

$$
\left\|v_{1}-\tau_{k^{i_{1}}} w_{i_{1}}\right\| \leq r \text {. }
$$

Therefore by Proposition 3.12 and (3.21), $v_{1}=\tau_{k^{i_{1}}} w_{i}$, and for large $m$

$$
\begin{aligned}
\frac{r}{8}-\varepsilon_{m} & \leq\left\|\sum_{i=2}^{k} \tau_{l_{m} n_{i}} v_{i}-\sum_{\substack{i=1 \\
i \neq i_{1}}}^{p} \tau_{k_{m}^{i}} w_{i}\right\| \\
& =\left\|\sum_{i=2}^{k} \tau_{l_{m}\left(n_{i}-n_{2}\right)} v_{i}-\sum_{\substack{i=1 \\
i \neq i_{1}}}^{p} \tau_{k_{m}^{i}-l_{m} n_{2}} w_{2}\right\| \leq r+\varepsilon_{m} .
\end{aligned}
$$

Thus we have returned to our original situation with one fewer term in each sum. Continuing in this fashion, we arrive at three possibilities depending on whether (a) $k>p$, (b) $k=p$, or (c) $k<p$. If (a) occurs, recalling the choice of $n_{0}$, for large $m$, we arrive at

$$
\frac{\nu}{2}<\left\|\sum_{i=p+1}^{k} \tau_{l_{m} n_{i}} v_{i}\right\| \leq r+\varepsilon_{m},
$$

contrary to (3.9) and (3.21). If (b) occurs, at the $k=p$ th step, we find

$$
x_{m}=\sum_{i=1}^{p} \tau_{k_{m}^{i}} w_{i},
$$

contrary to the lower bound of (3.28). Finally if (c) occurs, after $k$ steps for large $m$ we have

$$
\left\|\sum_{i=k+1}^{p} \tau_{k_{m}^{i}} w_{i}\right\| \leq r+\varepsilon_{m},
$$

which leads to a contradiction like (a) due to (1.27). 
Now our main existence assertions can be stated. Let $\lambda(t)$ and $\bar{\lambda}(t)$ denote respectively the smallest and largest eigenvalues of $L(t)$ and let

$$
\lambda=\min _{t \in[0, T]} \lambda(t), \quad \bar{\lambda}=\max _{t \in[0, T]} \bar{\lambda}(t) .
$$

By $\left(\mathrm{V}_{2}\right)$, there is a $\bar{\delta}>0$ such that $|x|,|y| \leq \bar{\delta}$ implies

$$
V(t, x) \leq(\lambda / 4)|x|^{2}
$$

and

$$
\left|V_{q}(t, x)-V_{q}(t, y)\right| \leq \frac{1}{2} \min (1, \lambda)|x-y| .
$$

The existence result that contains the most information but is somewhat awkward to state is:

Theorem 3.34. Let $r$ satisfy

$$
r<\min \left(r_{k}, \frac{1}{12} \mu\left(\mathscr{T}_{l}(\mathscr{F})\right), \nu / 2, \sqrt{\min \left(\frac{1}{2}, \lambda / 4\right)} \bar{\delta}\right)
$$

and let $n_{0}$ satisfy (3.9) and (3.16). Then $\left(\mathscr{K}_{k c-\alpha}^{k c+\alpha} / \mathbf{Z}\right) \cap \bar{N}_{r}(\mathscr{M}(k, l N, A)) \neq \varnothing$ for all but finitely many $l \in \mathbf{N}$.

An immediate consequence of Theorem 3.34 is

Corollary 3.36. If $V$ satisfies $\left(\mathrm{V}_{1}\right)-\left(\mathrm{V}_{3}\right)$ and $(*)$ holds, then for each $k \in$ $\mathbf{N} \backslash\{1\}, \mathscr{K}_{k c-\alpha}^{k c+\alpha} \backslash \mathbf{Z}$ is infinite.

Proof. We need only note that for any admissible $N$, the sets $\bar{N}_{r}(\mathscr{M}(k, \tilde{l} N, A))$ and $\bar{N}_{r}(\mathscr{M}(k, \bar{l} N, A))$ are disjoint if $|l-\tilde{l}|$ is sufficiently large.

Remark 3.37. Roughly speaking, Theorem 3.34 or Corollary 3.36 implies that for each $k \in \mathbf{N} \backslash\{1\}$, there is a solution $q$ of (HS) with $I(q)$ near $k c$, the bulk of the support of $q$ is concentrated in $k$ disjoint intervals, and the distance between adjacent intervals can be made as large as we please. This latter fact accounts for why there are infinitely many solutions in $\mathscr{K}_{k c-\alpha}^{k c+\alpha}$. Dynamically it means that the corresponding solutions start at the origin at $t=-\infty$, and return to a neighborhood of 0 where they remain for as long a time interval as we like, repeating this process $k-1$ times before terminating at 0 at $t=\infty$. Another consequence of Theorem 3.34 is a result for (HS) without assuming $(*)$.

Corollary 3.38. If $\left(\mathrm{V}_{1}\right)-\left(\mathrm{V}_{3}\right)$ and $(\mathrm{L})$ are satisfied, then there is an $\alpha>0$ such that $I^{2 c+\alpha} / \mathbf{Z}$ contains infinitely many distinct critical points of $I$.

Proof. If $(*)$ is false, $I^{c+\alpha} / \mathbf{Z}$ contains infinitely many critical points of $I$ for every $\alpha>0$, while if $(*)$ holds, Theorem 3.34 with $k=2$ gives the result.

Remark 3.39. This result is only of interest when $L$ or $V$ depend explicitly on $t$ due to the caveat made in the Introduction. 
The proof of Theorem 3.34 will be given in the next section.

\section{Proof of Theorem 3.34}

To prove Theorem 3.34, it suffices to show that $\mathscr{L}$ as defined in (3.22) is finite, for then (ii) of Proposition 3.20 must hold for all but finitely many $l \in \mathbf{N}$. A lengthy indirect argument will be employed to prove that $\mathscr{L}$ is finite. A brief sketch of it will be given now followed by the details.

Step 1. Using the fact that $\mathscr{L}$ is infinite, for an appropriate $\varepsilon=\varepsilon(r)$, we find $G \in \Gamma_{k}$ such that the $k$ components of the support of $G$ are separated by $k-1$ intervals of length $\beta$ with $\beta$ as large as we please and such that

$$
\max _{\theta \in[0,1]^{k}} I(G(\theta)) \leq k c+\varepsilon .
$$

Step 2. By an argument like the proof of Proposition 2.3, $G$ is deformed to $\bar{G}$ such that

$$
\max _{\theta \in[0,1]^{k}} I(\bar{G}(\theta)) \leq k c-\varepsilon .
$$

If $\bar{G} \in \Gamma_{k}$, we would have a contradiction to Proposition 3.5. Unfortunately we only know $\bar{G}$ is within $2 r$ of 0 in an $L^{\infty}$ sense on the $k-1$ intervals of length $\beta$.

Step 3. Using a variational problem on the " $\beta$ " intervals, we replace $\bar{G}$ on these intervals by $U$ such that $U$ is $L^{\infty}$ small compared to $\varepsilon$ near the center of each of these intervals and

$$
\max _{\theta \in[0,1]^{k}} I(U(\theta)) \leq k c-\varepsilon .
$$

Step 4. $U$ is modified near the center of each of the " $\beta$ " intervals obtaining $H \in \Gamma_{k}$ such that

$$
\max _{\theta \in[0,1]^{k}} I(H(\theta)) \leq k c-\frac{\varepsilon}{2} .
$$

This contradiction to Proposition 3.5 completes the proof.

Now for the details:

Step 1: Construction of $G$. Assuming that Theorem 3.34 is false and therefore $\mathscr{L}$ is infinite, let $\delta$ be given by Proposition 3.20. Choose

$$
\bar{\varepsilon}_{1}<\min \left(\frac{r \delta}{40}, \frac{\alpha_{1}}{2}\right),
$$

where $r$ satisfies (3.35) By Proposition 2.22 with this choice of $\bar{\varepsilon}_{1}, r_{1}=r / 16 k$, and $p=6 k$, there is an $\varepsilon=\varepsilon_{1} / 2 \in\left(0, \bar{\varepsilon}_{1}\right)$ and $g=g_{1} \in \Gamma$ such that

$$
\max _{\theta \in[0,1]} I(g(\theta)) \leq c+\frac{\varepsilon}{3 k}
$$

and

$$
I(g(\theta))>c-2 \varepsilon \quad \text { implies } \quad g(\theta) \in N_{r / 16 k}(A) .
$$


By a density argument as in Proposition 3.5, there is a $\hat{g} \in \Gamma$ and $R>0$ such that

$$
\begin{gathered}
\|g(\theta)-\hat{g}(\theta)\| \leq \frac{r}{16 k}, \\
|I(g(\theta))-I(\hat{g}(\theta))|<\frac{\varepsilon}{6 k}
\end{gathered}
$$

and

$$
\text { supp } \hat{g} \subset[-R, R]
$$

for all $\theta \in[0,1]$. By (4.5) and (4.2),

$$
\max _{\theta \in[0,1]} I(\hat{g}(\theta)) \leq c+\frac{\varepsilon}{2 k}
$$

Moreover if $I(\hat{g}(\theta))>c-3 \varepsilon / 2$, then by $(4.5)$

$$
I(g(\theta))=I(\hat{g}(\theta))+I(g(\theta))-I(\hat{g}(\theta))>c-\frac{3 \varepsilon}{2}-\frac{\varepsilon}{6 k}>c-2 \varepsilon .
$$

Thus by (4.3), $g(\theta) \in N_{r / 16 k}(A)$ and by (4.4), $\hat{g}(\theta) \in N_{r / 8 k}(A)$.

Now for $\theta \in[0,1]^{k}$, set

$$
G(\theta)=\sum_{i=1}^{k} \tau_{l n_{i}} \hat{g}\left(\theta_{i}\right),
$$

where $n_{0}$ satisfies (3.9) and (3.16) and $l$ is such that

$$
l T>2 R+\beta
$$

where $\beta>0$ and will be determined later in terms of $r$ and $L(t)$. By (4.8)(4.9) and the choice of $\hat{g}, G \in \Gamma_{k}$; by (4.7),

$$
I(G(\theta))=\sum_{i=1}^{k} I\left(\hat{g}\left(\theta_{i}\right)\right) \leq k c+\frac{\varepsilon}{2}<k c+\varepsilon
$$

and if $I(G(\theta))>k c-\varepsilon$, then

$$
I\left(\hat{g}\left(\theta_{i}\right)\right)+(k-1) c+\frac{(k-1) \varepsilon}{2 k}>k c-\varepsilon
$$

or

$$
I\left(\hat{g}\left(\theta_{i}\right)\right)>c-\frac{3 \varepsilon}{2}, \quad 1 \leq i \leq k
$$

Hence $\hat{g}\left(\theta_{i}\right) \in N_{r / 8 k}(A), 1 \leq i \leq k$, and

$$
G(\theta)=\sum_{i=1}^{k} \tau_{l n_{i}} \hat{g}\left(\theta_{i}\right) \in N_{r / 8}(\mathscr{M}(k, l N, A)) .
$$

Since $\mathscr{L}$ is infinite, for any given $\beta>0$, there is an $l \in \mathscr{L}$ satisfying (4.9) so that $I(G(\theta))>k c-\varepsilon$ implies $G(\theta) \in N_{r / 8}(\mathscr{W})$. This completes Step 1 . 
Step 2: The deformation of $G$. With the choices made above of $r, \varepsilon$, and $l$, consider the flow given by (2.7) for $x \in G(\theta)$, where $b=k c, \bar{\varepsilon}=\alpha$, and $\hat{\varepsilon} \in(\varepsilon, \bar{\varepsilon})$. If $I(G(\theta)) \leq k c-\varepsilon$ (which is the case if $G(\theta) \notin N_{r / 8}(\mathscr{W})$ ), we set $\sigma(G(\theta))=0$ so $\eta(\sigma(G(\theta)), G(\theta)) \in I^{k c-\varepsilon}$. Thus suppose $x=G(\theta)$ with $I(x)>k c-\varepsilon$. Then $x \in N_{r / 8}(\mathscr{W})$. If the solution $\eta(s, x)$ of $(2.7)$ does not exist for all $s \in[0,1]$, there is a maximal interval of existence $(0, \bar{s}(x))$ with $\bar{s}(x)<1$. If further $\eta(s, x)$ remains in $B_{r}(u)$ for some $u \in \mathscr{M}(k, l N, A)$, then if $s_{m} \rightarrow \bar{s}(x)$ and $u_{m}=\eta\left(s_{m}, x\right)$, by Proposition 1.24 there are functions $w_{i} \in \mathscr{K} \backslash\{0\}, 1 \leq i \leq p$, and sequences $\left(k_{m}^{i}\right), \quad 1 \leq i \leq p$, satisfying (1.27) such that

$$
\left\|u_{m}-\sum_{i=1}^{p} \tau_{k_{m}^{i}} w_{i}\right\| \rightarrow 0
$$

as $m \rightarrow \infty$. Taking the form of $u$ into account, by (4.13) we get

$$
\varlimsup_{m \rightarrow \infty}\left\|\sum_{i=1}^{k} \tau_{l n_{i}} v_{i}-\sum_{i=1}^{p} \tau_{k_{m}^{i}} w_{i}\right\| \leq r .
$$

If for some $i,\left(k_{m}^{i}\right)$ is bounded along a subsequence as $m \rightarrow \infty$, then by the argument of (3.27), there exists $z \in \bar{B}_{r}(u) \cap \mathscr{K}$, contrary to $l \in \mathscr{L}$. On the other hand, if $\left|k_{m}^{i}\right| \rightarrow \infty$ as $m \rightarrow \infty, 1 \leq i \leq p$, the argument of (3.26) yields a contradiction.

Thus either (i) $\eta(s, x)$ remains in $B_{r}(u)$ for $s \in[0,1]$, or (ii) $\eta(s, x)$ reaches $\partial B_{r}(u)$ at some $s \leq 1$. If (i), the arguments of Proposition 2.3 show there is a continuous $\sigma(x)<1$ such that

$$
I(\eta(\sigma(G(\theta)), G(\theta)))=k c-\varepsilon .
$$

If (ii) occurs, $\eta(s, x)$ must cross from $B_{r / 8}(u)$ to $\partial B_{r}(u)$. If in this time interval $\eta(s, x)$ did not reach $\partial I^{k c-\varepsilon}$, then, as in (2.9)-(2.10), (2.13)-(2.14) (using Proposition 3.20),

$$
\frac{7}{8} r \leq \frac{4 \varepsilon}{\delta} \quad \text { or } \quad \frac{7}{32} r \delta<\varepsilon,
$$

contrary to (4.1) and $\varepsilon<\bar{\varepsilon}_{2}$. Thus as with (i), there is a $\sigma(x)<1$ such that (4.15) holds. Moreover, as in the proof of Proposition 2.3,

$$
\|\eta(\sigma(G(\theta)), G(\theta))-G(\theta)\| \leq r .
$$

Remark 4.17. If $\bar{G}(\theta) \equiv \eta(\sigma(G(\theta)), G(\theta)) \in \Gamma_{k}$, (4.15) would provide a contradiction to Proposition 3.5. By (4.16),

$$
\|\bar{G}(\theta)-G(\theta)\|_{L^{\infty}} \leq 2 r
$$

and, in particular,

$$
|\bar{G}(\theta)(t)| \leq 2 r
$$


for $t$ in the intervals where $G(\theta)$ vanishes: $[R, R+\beta]$, etc. Since $r$ is small, it is tempting to try to truncate $\bar{G}(t)$ in the intervals $[R, R+\beta]$, etc. to obtain $H \in \Gamma_{k}$. However the error made in this process seems to go like $r$ which is large compared to $\varepsilon$ and therefore it cannot be guaranteed that $I(H)<k c$. Alternatively since $\beta$ is large and (4.16) holds, for each $\theta$ there are points $t \in[R, R+\beta]$ where $|\bar{G}(\theta)(t)|$ can be made small compared to $\varepsilon$ and $\bar{G}(\theta)$ can be truncated at such points. However we do not know how to make a continuous selection with respect to $\theta \in[0,1]^{k}$. Thus a more complicated procedure will be followed in Step 3.

Before going on to Step 3, some observations are needed. Note that for $1 \leq i \leq k$,

$$
G\left(0_{i}\right)=\sum_{j \neq i} \tau_{l n_{j}} \hat{g}\left(\theta_{j}\right)
$$

and

$$
\begin{aligned}
I\left(G\left(0_{i}\right)\right) & =\sum_{j \neq i} I\left(\hat{g}\left(\theta_{j}\right)\right) \leq(k-1)\left(c+\frac{\varepsilon}{2 k}\right) \\
& \leq(k-1) c+\frac{\varepsilon}{2}<k c-\varepsilon
\end{aligned}
$$

since $\varepsilon<\alpha<c / 2$. Thus, as in Proposition 2.3, $\sigma\left(G\left(0_{i}\right)\right)=0$ and

$$
\bar{G}\left(0_{i}\right)=G\left(0_{i}\right), \quad 1 \leq i \leq k .
$$

Similarly

$$
G\left(1_{i}\right)=\tau_{\ln _{i}} \hat{g}(1)+\sum_{j \neq i} \tau_{\ln j} \hat{g}\left(\theta_{j}\right)
$$

so $I\left(G\left(1_{i}\right)\right)<k c-\varepsilon, \sigma\left(G\left(1_{i}\right)\right)=0$, and

$$
\bar{G}\left(1_{i}\right)=G\left(1_{i}\right), \quad i \leq i \leq k .
$$

Step 3: The construction of $U(\theta)$. $U(\theta)$ will be obtained from $\bar{G}(\theta)$ by redefining $\bar{G}(\theta)$ in an appropriate way in the $k-1$ intervals $[R, R+\beta]$, etc. where $G(\theta)$ vanishes. Let $x \in W^{1,2}\left([R, R+\beta], \mathbf{R}^{n}\right) \equiv Y$ and set

$$
\Psi(x)=\int_{R}^{R+\beta}\left[\frac{1}{2}\left(|\dot{x}|^{2}+L(t) x \cdot x\right)-V(t, x)\right] d t .
$$

Then $\Psi$ is $C^{1}$ on this class of functions and is also weakly lower semicontinuous. Note also that $\Psi$ is not bounded from above or below. However if $\Psi$ is restricted to the subclass of $x \in W^{1,2}\left([R, R+\beta], \mathbf{R}^{n}\right)$ such that $x(R)=0=x(R+\beta)$, then $x=0$ is a strict local minimum for $\Psi$. This suggests that $\Psi$ should also have a small strict local minimum for any other subclass of functions which are small at $R, R+\beta$. It is this fact that will be exploited to construct the modification of $\bar{G}$.

Let

$$
S=\bigcup_{i=0}^{k-2}[(2 i+1) R+i \beta,(2 i+1) R+(i+1) \beta]
$$


We use (4.22) to extend $\Psi$ to $S$ in the obvious fashion. For each $\theta \in[0,1]^{k}$, define

$$
U(\theta)(t)=\bar{G}(\theta)(t), \quad t \notin S,
$$

while for $t \in S, U(\theta)(t)$ is the solution of the variational problem

$$
\text { minimize } \Psi(x),
$$

where the minimum is taken over the set of $x \in W^{1,2}\left(S, \mathbf{R}^{n}\right)$ such that $x(t)=$ $\bar{G}(\theta)(t)$ for $t \in \partial S$ and $\|x\|_{L^{\infty}\left(S, \mathbf{R}^{n}\right)} \leq \bar{\delta}$. Here $\bar{\delta}$ is as defined in (3.32)-(3.33). Thus (4.19) shows $\left.\bar{G}(\theta)\right|_{S}$ lies in the above class of functions.

Remark 4.25. It is clear that, if it exists, the minimizer restricted to a component of $S$ minimizes $\Psi$ on the corresponding class of functions with $S$ replaced by the component of $S$.

Proposition 4.26. There exists a unique solution, $\bar{x}$, of (4.24). Furthermore $\bar{x}$ depends continuously on $\theta \in[0,1]^{k}$.

Proof. By Remark 4.25, it suffices to work with $[R, R+\beta]$. The choice of $\bar{\delta}$ shows that, for admissible $x$,

$$
\begin{aligned}
\Psi(x) & \geq \int_{R}^{R+\beta}\left(\frac{1}{2}\left(|\dot{x}|^{2}+L(t) x \cdot x\right)-\frac{\lambda}{4}|x|^{2}\right) d t \\
& \geq \min \left(\frac{1}{2}, \frac{\lambda}{4}\right)\|x\|_{Y}^{2} .
\end{aligned}
$$

Let $\left(x_{m}\right)$ be a minimizing sequence for (4.24). Then by (4.27), for $m$ large

$$
\begin{aligned}
\min \left(\frac{1}{2}, \frac{\lambda}{4}\right)\left\|x_{m}\right\|_{Y}^{2} & \leq \Psi\left(x_{m}\right) \leq \Psi(\bar{G}(\theta)) \\
& \leq \int_{R}^{R+\beta}\left[\frac{1}{2}\left(|\dot{\bar{G}}(\theta)|^{2}+L(t) \bar{G}(\theta) \cdot \bar{G}(\theta)\right)\right] d t
\end{aligned}
$$

By (4.16),

$$
\left(\int_{R}^{R+\beta}|\dot{\bar{G}}(\theta)|^{2}+L(t) \bar{G}(\theta) \cdot \bar{G}(\theta) d t\right)^{1 / 2} \leq\|\bar{G}(\theta)-G(\theta)\| \leq r .
$$

Hence (4.28)-(4.29) imply

$$
\left\|x_{m}\right\|_{Y}^{2} \leq \frac{1 / 2}{\min (1 / 2, \lambda / 4)} r^{2}
$$

Since for all $x \in E$,

$$
\|x\|_{L^{\infty}\left([R, R+\beta], \mathbf{R}^{n}\right)} \leq \sqrt{2}\|x\|_{Y}
$$

by (4.30)-(4.31) and (3.35), $\left\|x_{m}\right\|_{L^{\infty}\left([R, R+\beta], \mathbf{R}^{n}\right)}<\bar{\delta}$. Thus, for $m$ large, the minimizing sequence automatically satisfies the $L^{\infty}$ constraint. By (4.30)-(4.31), a subsequence of $\left(x_{m}\right)$ converges weakly in $Y$ and strongly in 
$L^{\infty}\left([R, R+\beta], \mathbf{R}^{n}\right)$ to $\bar{x}$ satisfying $\|\bar{x}\|_{L^{\infty}\left([R, R+\beta], \mathbf{R}^{n}\right)}<\bar{\delta}$. Standard arguments from the theory of elliptic boundary value problems then show $\bar{x}$ minimizes $\Psi$ and is a classical solution of

$$
\begin{cases}\ddot{x}-L(t) x+V_{q}(t, x)=0, & t \in(R, R+\beta), \\ x(t)=\bar{G}(\theta)(t), & t=R, R+\beta .\end{cases}
$$

We claim $\bar{x}$ is the unique solution of (4.32) satisfying the $L^{\infty}$ constraint. Indeed if there were two such solutions $x, y$, then by (3.33),

$$
\begin{aligned}
\min (1, \lambda)\|x-y\|_{Y}^{2} & \leq \int_{R}^{R+\beta}\left[|\dot{x}-\dot{y}|^{2}+L(t)(x-y) \cdot(x-y)\right] d t \\
& =\int_{R}^{R+\beta}\left(V_{q}(t, x)-V_{q}(t, y)\right) \cdot(x-y) d t \\
& \leq \frac{1}{2} \min (1, \lambda)\|x-y\|_{Y}^{2},
\end{aligned}
$$

which implies $x=y$. Finally the continuity of $\bar{x}$ is immediate from the uniqueness of $\bar{x}$.

With $U(\theta)$ now defined, note that, by construction,

$$
I(U(\theta)) \leq I(\bar{G}(\theta)) \leq k c-\varepsilon .
$$

We also have to keep track of $U\left(0_{i}\right)$ and $U\left(1_{i}\right)$. For $t \notin S$, by (4.20) and (4.23) for $1 \leq i \leq k$,

$$
U\left(0_{i}\right)(t)=G\left(0_{i}\right)(t) .
$$

Since $G\left(0_{i}\right)(t)=0$ for $t \in S,(4.32)$ and the uniqueness assertion of Proposition 4.26 imply $U\left(0_{i}\right)(t)=0=G\left(0_{i}\right)(t)$ for $t \in S$ and therefore

$$
U\left(0_{i}\right)=G\left(0_{i}\right), \quad 1 \leq i \leq k .
$$

Similarly, by (4.21) and (4.23), for $t \notin S$,

$$
U\left(1_{i}\right)(t)=G\left(1_{i}\right)(t)
$$

and $G\left(1_{i}\right)(t)=0$ for $t \in S$, so as above,

$$
U\left(1_{i}\right)=G\left(1_{i}\right), \quad 1 \leq i \leq k .
$$

To continue, estimates are needed for $U(\theta)(t)$ for $t$ near the center of each of the subintervals that make up $S$. We will show that if $\beta$ is sufficiently large, then $U(\theta)(t)$ is small (compared to $\varepsilon$ ) and this will allow us to redefine $U$ to get $H \in \Gamma_{k}$ in Step 4. A comparison argument will be used to get the necessary estimate.

Let

$$
\bar{L}(t) \equiv-\frac{d^{2}}{d t^{2}}+\gamma,
$$

where the positive constant $\gamma$ is free for the moment. Let $w=w(\theta)(t)$ satisfy

$$
\left\{\begin{array}{l}
\bar{L} w=0 \quad \text { in } S, \\
w=\max _{t \in \partial S}|U(\theta)(t)| \equiv a, \quad t \in \partial S .
\end{array}\right.
$$


Note that for $t \in S$, by (3.33),

$$
\begin{aligned}
\bar{L}|\bar{x}|^{2} & =-2|\dot{\bar{x}}|^{2}-2 \bar{x} \cdot \ddot{\bar{x}}+\gamma|\bar{x}|^{2} \\
& \leq-2|\dot{\bar{x}}|^{2}-2 L(t) \bar{x} \cdot \bar{x}+\min (1, \lambda)|\bar{x}|^{2}+\gamma|\bar{x}|^{2} .
\end{aligned}
$$

Choose $\gamma=2 \lambda$. Then

$$
\bar{L}|\bar{x}|^{2} \leq 0 \quad \text { in } S
$$

and

$$
\begin{cases}\bar{L}\left(w-|\bar{x}|^{2}\right) \geq 0 & \text { on } S \\ w-|\bar{x}|^{2} \geq 0 & \text { on } \partial S .\end{cases}
$$

Consequently, by the Maximum Principle,

$$
w(t) \geq|\bar{x}(t)|^{2}, \quad t \in S .
$$

Now $w$ can be written down explicitly in each of the subintervals that make up $S$; e.g., for $t \in[R, R+\beta]$,

$$
w(t)=a\left(\frac{1-e^{-\sqrt{\gamma} \beta}}{e^{\sqrt{\gamma} \beta}-e^{-\sqrt{\gamma} \beta}}\right) e^{\sqrt{\gamma}(t-R)}+a\left(\frac{e^{\sqrt{\gamma} \beta}-1}{e^{\sqrt{\gamma} \beta}-e^{-\sqrt{\gamma} \beta}}\right) e^{-\sqrt{\gamma}(t-R)} .
$$

By (4.44)-(4.45) for $s \in\left[-\frac{1}{2}, \frac{1}{2}\right]$ we have

$$
\left|\bar{x}\left(R+\frac{\beta}{2}+s\right)\right|^{2} \leq w\left(R+\frac{\beta}{2}+s\right) \leq 2 a e^{\sqrt{\gamma}(1-\beta) / 2} .
$$

Thus $\bar{x}$ is exponentially small (in $\beta$ ) in $\left[R+\frac{\beta}{2}-\frac{1}{2}, R+\frac{\beta}{2}+\frac{1}{2}\right]$ with similar estimates for the remaining subintervals of $S$.

Step 4: The construction of $H$. Define

$$
H(\theta)(t)=U(\theta)(t),
$$

where

$$
\begin{aligned}
& t \notin S_{1} \equiv {\left[R+\frac{\beta}{2}-\frac{1}{2}, R+\frac{\beta}{2}+\frac{1}{2}\right] } \\
& \cup\left[3 R+\frac{3 \beta}{2}-\frac{1}{2}, 3 R+\frac{3 \beta}{2}+\frac{1}{2}\right] \cup \cdots, \\
& H(\theta)\left(R+\frac{\beta}{2}\right)=0=H(\theta)\left(3 R+\frac{3 \beta}{2}\right)=\cdots,
\end{aligned}
$$

and $H(\theta)(t)$ is interpolated linearly for the remaining regions. We claim $H \in$ $\Gamma_{k}$. To verify $\left(\mathrm{g}_{1}\right)-\left(\mathrm{g}_{2}\right)$, note that by $(4.47)$, for $1 \leq i \leq k$

$$
H\left(0_{i}\right)(t)=U\left(0_{i}\right)(t), \quad t \notin S_{1} .
$$

Since $U\left(0_{i}\right)(t)=0$ on $\partial S_{1},(4.47)$ implies $H\left(0_{i}\right)(t)=0, t \in S_{1}$, and therefore

$$
H\left(0_{i}\right)=G\left(0_{i}\right), \quad 1 \leq i \leq k,
$$


via (4.36). Similarly

$$
H\left(1_{i}\right)=G\left(1_{i}\right), \quad 1 \leq i \leq k .
$$

Defining $p_{0}=-\infty, p_{k}=\infty, p_{1}=R+\frac{\beta}{2}, p_{2}=3 R+\frac{3 \beta}{2}$, etc., and

$$
h_{i}(\theta)(t)= \begin{cases}H(\theta)(t) & \text { for } t \in\left[p_{i-1}, p_{i}\right], \\ 0 & \text { otherwise }\end{cases}
$$

we see $H \in \Gamma_{k}$.

We claim that for $\beta$ sufficiently large,

$$
I(H(\theta)) \leq k c-\frac{\varepsilon}{2},
$$

contrary to Proposition 3.5. Indeed,

$$
\begin{aligned}
I(H(\theta)) & =I(U(\theta))+I(H(\theta))-I(U(\theta)) \\
& \leq k c-\varepsilon+I(H(\theta))-I(U(\theta)) .
\end{aligned}
$$

Thus to verify (4.51), it suffices to prove

$$
I(H(\theta))-I(U(\theta)) \leq \frac{\varepsilon}{2} .
$$

By the definition of $H$, the left-hand side of (4.53) is the sum of $k-1$ terms, the first of which is

$$
\begin{aligned}
\int_{R+\frac{\beta}{2}-\frac{1}{2}}^{R+\frac{\beta}{2}+\frac{1}{2}} & {\left[\frac{1}{2}\left(|\dot{H}(\theta)|^{2}+L(t) H(\theta) \cdot H(\theta)\right)-V(t, H(\theta))\right.} \\
& \left.-\frac{1}{2}\left(|\dot{U}(\theta)|^{2}+L(t) U(\theta) \cdot U(\theta)\right)+V(t, U(\theta))\right] d t
\end{aligned}
$$

We will show that for $\beta$ sufficiently large, each of the $H$ and $U$ contributions to (4.54) is less than $\varepsilon / 4 k$. To estimate the $U$ contribution, note first from (4.32), (4.46), and (3.33), for $t \in\left[R+\frac{\beta}{2}-\frac{1}{2}, R+\frac{\beta}{2}+\frac{1}{2}\right]$,

$$
\begin{aligned}
|\ddot{\bar{x}}(t)| & \leq|L(t) \bar{x}|+\left|V_{q}(t, \bar{x})\right| \leq\left(\|L\|_{L^{\infty}}+\frac{1}{2}(1+\lambda)\right)|\bar{x}(t)| \\
& \leq \sqrt{2 a}\left(\|L\|_{L^{\infty}}+\frac{1}{2}(1+\lambda)\right) e^{\sqrt{\gamma}(1-\beta) / 4} \equiv M_{1} e^{-\gamma_{1} \beta},
\end{aligned}
$$

where $\gamma_{1}=\frac{1}{4} \sqrt{\gamma}$ and $M_{1}$ depends on $\lambda,\|L\|_{L^{\infty}}$, and $r$. (Recall that $a$ depends on $r$.) Since

$$
\dot{\bar{x}}(t)=\dot{\bar{x}}(s)+\int_{s}^{t} \ddot{\bar{x}}(\xi) d \xi,
$$

integrating (4.56) over $s \in\left[R+\frac{\beta}{2}-\frac{1}{2}, R+\frac{\beta}{2}+\frac{1}{2}\right]$ yields

$$
\begin{aligned}
|\dot{\bar{x}}(t)| \leq & \left|\bar{x}\left(R+\frac{\beta}{2}+\frac{1}{2}\right)\right|+\left|\bar{x}\left(R+\frac{\beta}{2}-\frac{1}{2}\right)\right| \\
& +\|\ddot{\bar{x}}\|_{L^{\infty}\left(\left[R+\frac{\beta}{2}-\frac{1}{2}, R+\frac{\beta}{2}+\frac{1}{2}\right], \mathbf{R}^{n}\right)} \\
\leq & M_{2} e^{-\gamma_{1} \beta}
\end{aligned}
$$


where

$$
M_{2}=2 \sqrt{2 a} e^{\gamma_{1}}+M_{1} .
$$

Thus by (4.46), (4.57), and (3.32),

$$
\left|\int_{R+\frac{\beta}{2}-\frac{1}{2}}^{R+\frac{\beta}{2}+\frac{1}{2}}\left[\frac{1}{2}\left(|\dot{U}|^{2}+L(t) U \cdot U\right)-V(t, U)\right] d t\right| \leq M_{3} e^{-2 \gamma_{1} \beta}
$$

where

$$
M_{3}=\frac{1}{2}\left[M_{2}^{2}+2 a e^{\sqrt{\gamma} / 2}\left(\|L\|_{L^{\infty}}+\lambda / 2\right)\right] .
$$

Similarly by (4.47), (4.57), and (4.46), for $t \in\left[R+\frac{\beta}{2}-\frac{1}{2}, R+\frac{\beta}{2}+\frac{1}{2}\right]$,

$$
|\dot{H}(\theta)(t)| \leq M_{2} e^{-\gamma_{1} \beta}, \quad|H(\theta)(t)| \leq \sqrt{2 a} e^{\gamma_{1}(1-\beta)}
$$

and therefore

$$
\left|\int_{R+\frac{\beta}{2}-\frac{1}{2}}^{R+\frac{\beta}{2}+\frac{1}{2}}\left(\frac{1}{2}\left(|\dot{H}|^{2}+L(t) H \cdot H\right)-V(t, H)\right) d t\right| \leq M_{3} e^{-2 \gamma_{1} \beta}
$$

It follows that

$$
I(H(\theta))-I(U(\theta)) \leq 2(k-1) M_{3} e^{-2 \gamma_{1} \beta} \leq \frac{\varepsilon}{2}
$$

provided $\beta=\beta\left(r, \lambda,\|L\|_{L^{\infty}}\right)$ is sufficiently large. Then (4.51) holds and Theorem 3.34 is proved.

\section{ON WEAKENING $(*)$}

We conjecture that Theorem 3.34 and Corollary 3.36 remain valid if $(*)$ is replaced by the milder assumption:

(**) There is an $\alpha>0$ such that $\mathscr{K}^{c+\alpha}$ consists of isolated points.

In this section, we will indicate why this conjecture is true if an additional assumption is made on $V$. In fact an even weaker condition than (**) will then be required.

To begin, observe that if $q$ is a solution of (HS), then

$$
\|q\|^{2}=\int_{-\infty}^{\infty} V_{q}(t, q) \cdot q d t
$$

Let

$$
\mathscr{S} \equiv\{q \in E \backslash\{0\} \mid q \text { satisfies (5.1) }\}
$$

Thus $\mathscr{K} \backslash\{0\} \subset \mathscr{S}$. For $\rho>0$ and $q \in E \backslash\{0\}$, let $\psi(\rho)=I(\rho q)$. At a nonzero critical point of $\psi$,

$$
\rho\|q\|^{2}=\int_{-\infty}^{\infty} V_{q}(t, \rho q) \cdot q d t .
$$

Multiplying (5.3) by $\rho$ shows $\rho q \in \mathscr{S}$. Suppose $V$ satisfies $\left(\mathrm{V}_{4}\right)$ for all $\xi \in S^{n-1}, \rho^{-1} V_{q}(t, \rho \xi) \cdot \xi$ is an increasing function of $\rho$. 
Then

$$
\psi^{\prime}(\rho)=\rho\left(\|q\|^{2}-\rho^{-1} \int_{-\infty}^{\infty} V_{q}(t, \rho q) \cdot q d t\right) .
$$

By $\left(\mathrm{V}_{2}\right)$, as $\rho \rightarrow 0$,

$$
\rho^{-1} \int_{-\infty}^{\infty} V_{q}(t, \rho q) \cdot q d t \rightarrow 0
$$

and by $\left(\mathrm{V}_{3}\right)$, as $\rho \rightarrow \infty$,

$$
\rho^{-1} \int_{-\infty}^{\infty} V_{q}(t, \rho q) q d t \rightarrow \infty .
$$

Consequently, by $\left(\mathrm{V}_{4}\right)$ and $(5.4)-(5.6)$, there is a unique $\bar{\rho}=\bar{\rho}(q)>0$ such that $\psi^{\prime}(\bar{\rho})=0$ and

$$
\psi(\bar{\rho})=\max _{\rho \geq 0} I(\rho q) .
$$

Let $y \in E \backslash\{0\}$ such that $I(y)<0$. Then $g_{y}(\theta) \equiv \theta y \in \Gamma$. Therefore

$$
0<c=\inf _{g \in \Gamma} \max _{\theta \in[0,1]} I(g(\theta)) \leq \inf _{y \in\{y \mid I(y)<0\}} \max _{\theta \in[0,1]} I\left(g_{y}(\theta)\right) .
$$

By above remarks, $\max I\left(g_{y}(\theta)\right)$ occurs on $\mathscr{S}$. Hence by $(5.8)$

$$
0<c \leq \inf _{\mathscr{S}} I .
$$

On the other hand, if $q \in \mathscr{K} \backslash\{0\}$, then $q \in \mathscr{S}$ and, e.g., by [1], $\left(\mathrm{V}_{1}\right)-\left(\mathrm{V}_{3}\right)$ imply $\mathscr{K} \backslash\{0\} \neq \varnothing$. Therefore

$$
c \leq \inf _{\mathscr{K} \backslash\{0\}} I \equiv \bar{c} .
$$

We claim $c$ is a critical value of $I$. If not, $c<\bar{c}$ and therefore (*) holds. Hence by Theorem $2.19, c$ is a critical value of $I$. Thus

$$
c=\inf _{\mathscr{S}} I=\inf _{\mathscr{K} \backslash\{0\}} I .
$$

Let $v$ be a critical point of $I$. Choosing $y$ on the ray passing through 0 and $v$ so that $I(y)<0$, we have

$$
\max _{\theta \in[0,1]} I\left(g_{y}(\theta)\right)=c
$$

so $g_{y}$ satisfies $1^{\circ}$ of Proposition 2.22 with $\varepsilon_{1}=0$. Let $\bar{\varepsilon}_{1}$ be as in Proposition 2.22 and suppose $v=\bar{\theta} y$. For any $\varepsilon_{1}<\bar{\varepsilon}_{1}$, by $\left(\mathrm{V}_{4}\right)$, there are $\theta_{-}\left(\varepsilon_{1}\right)<\bar{\theta}<$ $\theta_{+}\left(\varepsilon_{1}\right)$ such that $\theta_{ \pm}\left(\varepsilon_{1}\right) \rightarrow 0$ as $\varepsilon_{1} \rightarrow 0$ and $I(\theta y)>c-\varepsilon_{1}$ for $\theta \in\left(\theta_{-}, \theta_{+}\right)$. In particular, for any given $r_{1}>0$, there is an $\varepsilon_{1}\left(r_{1}\right)$ such that $\theta \in\left(\theta_{-}, \theta_{+}\right)$ implies $\theta y \in B_{r_{1}}(v)$. Hence $2^{\circ}$ of Proposition 2.22 holds with this $r_{1}$ and $A=\{v\}$.

Thus having $\left(\mathrm{V}_{4}\right)$ we are able to bypass the construction of Proposition 2.22 to find $g=g_{y}$ as above and $A=\{v\}$. Now $\mathscr{M}(k, N, A)$ and $\mathscr{M}^{*}(k, N, A)$ can be defined as in (3.10)-(3.11). By $(* *)$, there is an $r^{*}>0$ such that

$$
B_{r^{*}}(v) \cap \mathscr{K}=\{v\} .
$$


Let

$$
r<\min \left(\frac{1}{12} \mu(\mathscr{T}(A)), \nu / 2, r_{k}, r^{*}\right) .
$$

(Note that $\bar{l}=1$ here.) An examination of the proof of Proposition 3.20 shows the only places $(*)$ enters is via $\mu(\mathscr{T}(\mathscr{F}))$, which we replace by $\mu(\mathscr{T}(A))$ and in the argument following (3.29). Choosing $r$ to satisfy (5.14) shows Proposition 3.20 is still valid. Likewise adding $r^{*}$ to the right-hand side of $(3.35)$, the proof of Theorem 3.34 and Corollary 3.36 carry over to our current situation giving us infinitely many solutions of (HS) in $\left(\mathscr{K}_{k c-\alpha}^{k c+\alpha} / \mathbf{Z}\right) \cap \bar{N}_{r}\left(\mathscr{M}^{*}(k, N, A)\right)$. In fact we do not need $(* *)$ but simply that $v$ is an isolated point in $\mathscr{K}(c)$.

\section{ACKNOWLEDGMENT}

Part of this work was done while the first author was visiting the Center for the Mathematical Sciences of the University of Wisconsin. He wants to thank P. Rabinowitz and all the faculty and staff of CMS.

\section{REFERENCES}

1. P. H. Rabinowitz, Homoclinic orbits for a class of Hamiltonian systems, Proc. Roy. Soc. Edinburgh Sect. A 114 (1990), 33-38.

2. V. Coti Zelati, I. Ekeland, and E. Séré, A variational approach to homoclinic orbits in Hamiltonian systems, Math. Ann. 288 (1990), 133-160.

3. E. Séré, Une approche variationnelle au problème des orbites homoclines de systèmes hamiltonian, Math. Z., to appear.

4. H. Hofer and K. Wysocki, First order elliptic systems and the existence of homoclinic orbits in Hamiltonian systems, preprint.

5. K. Tanaka, Homoclinic orbits in a first order superquadratic Hamiltonian system: Convergence of subharmonic orbits, Analyse nonlinéaire, to appear.

6. P. H. Rabinowitz, Minimax methods in critical point theory with applications to differential equations, CBMS Regional Conf. Ser. in Math., no. 65, Conf. Board Math. Sci., Washington, D.C., 1986.

SISSA, 34014 TRIESTE, ITALY

Department of Mathematics and Center for the Mathematical Sciences, University of Wisconsin, MADISON, Wisconsin 53706 